\title{
Aproximación multivariante en la exploración de la tolerancia ambiental de las familias de macroinvertebrados de los ríos mediterráneos del proyecto GUADALMED.
}

\author{
Soledad Vivas ${ }^{1}$, Jesús Casas ${ }^{1}$, Isabel Pardo ${ }^{2}$, Santiago Robles $^{3}$, Núria Bonada ${ }^{4}$, Andrés Mellado ${ }^{5}$, \\ Narcís Prat ${ }^{4}$, Javier Alba-Tercedor ${ }^{6}$, Maruxa Álvarez ${ }^{2}, \mathrm{M}^{\mathrm{a}}$ del Mar Bayo ${ }^{1}$, Pablo Jáimez-Cuéllar ${ }^{6}, \mathrm{M}^{\mathrm{a}}$ \\ Luisa Suárez ${ }^{5}$, Manuel Toro ${ }^{3}, \mathrm{M}^{\mathrm{a}}$ Rosario Vidal-Abarca ${ }^{5}$, Carmen Zamora-Muñoz ${ }^{6}$ y Gabriel Moyá ${ }^{7}$ \\ ${ }^{1}$ Departamento de Biología Vegetal y Ecología. Universidad de Almería. Cañada de San Urbano, s/n. 04120 \\ Almería. \\ 2 Área de Ecología. Universidad de Vigo. Campus Lagoas-Marcosende. 36200 Vigo. \\ ${ }^{3}$ CEDEX. División de Ecología de los Sistemas Acuáticos Continentales. Paseo Bajo Virgen del Puerto, 3. \\ 28005 Madrid. \\ ${ }^{4}$ Departament d'Ecologia. Universitat de Barcelona. Diagonal, 645. 08028 Barcelona. \\ ${ }^{5}$ Departamento de Ecología e Hidrología. Universidad de Murcia. Campus de Espinardo. 30100 Murcia. \\ ${ }^{6}$ Departamento de Biología Animal y Ecología. Universidad de Granada. Campus Universitario de \\ Fuentenueva. 18071 Granada. \\ ${ }^{7}$ Departament de Biologia. Universitat de les Illes Balears. Crta. Valldemosa, km. 7.5. 07071 Palma de \\ Mallorca.
}

\section{RESUMEN}

Se han estudiado los macroinvertebrados y algunas características físico-químicas y fisiográficas (temperatura, $\mathrm{pH}$, área de la cuenca, orden del río, altitud, caudal, conductividad, amonio, nitritos, nitratos, fosfatos) en 157 localidades distribuidas en 12 cuencas fluviales mediterráneas de la Peninsula Ibérica e isla de Mallorca. Ciento veintisiete taxones, con una resolución taxonómica en su mayoría a familia, fueron censados en el total de las cuencas. La exploración de tendencias de variación de las familias y su relación con las variables físico-químicas del agua se realizó mediante dos tipos de análisis de gradiente: análisis de correspondencias (AC) y análisis canónico de correspondencias (ACC). Las variables fisico-químicas seleccionadas (ACC) explicaron algo más de la mitad de la pura varianza de la matriz de familias (AC) absorbida por las 3 primeras dimensiones canónicas. Salinidad y temperatura resultaron las principales variables que condicionan la distribución de las familias. Estas determinan un gradiente ambiental, de génesis principalmente natural, que se relaciona negativamente con altitud y caudal. Las familias más halo-termófilas, según esta ordenación, fueron Thiaridae y Ephydridae, y en general, crustáceos, odonatos, coleópteros y heterópteros $(\mathrm{OCH})$. Los plecópteros se mostraron los más estenoicos hacia el extremo bajo de este gradiente, seguidos de efemerópteros y tricópteros. El caudal generó un segundo gradiente ambiental que se puede relacionar también con la temporalidad del medio acuático, aunque aquí resulta difícil deslindar el régimen natural de las perturbaciones antrópicas. La contaminación orgánica representa un tercer gradiente, en el que las familias Asellidae, Syrphidae, Chironomidae y Physidae se decantan por el enriquecimiento orgánico. En general, la riqueza de familias se mostró muy negativamente condicionada por la contaminación. La mayor correlación negativa del índice biótico IASPT con el gradiente salinidad-termicidad en comparación con el de contaminación, advierte de la cautela con la que debemos interpretar los resultados proporcionados por este índice. En esta flaqueza subyace la tendencia general a sobrestimar, en términos de valor indicador de calidad, al grupo taxonómico EPT frente al OCH. El índice IBMWP disimula este sesgo al contar con la riqueza de familias, ya que esta variable no se correlacionó con el gradiente salinidad-termicidad.

Palabras clave: familias de macroinvertebrados, ríos mediterráneos, gradientes ambientales, tolerancia ambiental, índices bióticos.

\begin{abstract}
Macroinvertebrates and selected physico-chemical characteristics (temperature, pH, basin area, stream order, altitude, discharge, conductivity, ammonium, nitrites, nitrates, phosphates) were studied in 157 sites distributed over 12 Mediterranean fluvial basins located in the Iberian Peninsula and in the island of Majorca. A total of 127 taxa were recorded, mostly identi-
\end{abstract}


fied to the taxonomic resolution of family. Correspondence analysis (CA) and canonical correspondence analysis (CCA) were used to explore the tendencies of variation of the abundances of different families and their relationships with physico-chemical variables. The selected environmental variables explained more than half of the pure variance of the data matrix of families on the three first canonical axes. Salinity and temperature were the main variables explaining the distribution of families. These variables formed a natural gradient which was negatively correlated with altitude and discharge. The most halo-thermophilous families, according to this ordination, were Thiaridae and Ephydridae, followed by crustaceans, Odonata, Coleoptera and Heteroptera $(\mathrm{OCH})$. Stoneflies were the group of families favouring narrow ranges of the lower values of temperature and salinity, followed by mayflies and caddisflies. A second environmental gradient was chiefly correlated with discharge. However, the gradient was also related with temporality of the aquatic habitat. In this second gradient it was difficult to separate the natural regime from anthropogenic disturbances. Organic pollution was associated with the third emerging gradient. Asellidae, Syrphidae, Chironomidae and Physidae were the macroinvertebrate families most related to levels of organic enrichment. Generally, the richness of families whithin any taxonomic group, showed very strong negative correlations with the organic pollution-associated gradient. The IASPT biotic index showed a stronger negative correlation with the gradient salinity-temperature than with the pollution gradient. Therefore, biotic assessments using this index must by done cautiously. In this weakness underlies the general tendency to overestimate the indicator value of good quality attributed to the families in the group EPT compared to that of the orders OCH. The IBMWP biotic index was, in great part, immune to the above bias thanks to the major contribution of family richness in the calculation of the index. Family richness was not correlated to the gradient salinity-temperature.

Keywords: Macroinvertebrate families, Mediterranean streams, environmental gradients, environmental tolerance, biotic indices

\section{INTRODUCCIÓN}

Los estudios sobre comunidades fluviales de macroinvertebrados bentónicos en la vertiente mediterránea de la Península Ibérica, y en general de toda la Península e Islas Baleares, se iniciaron decididamente en la década de los 80 y se prodigaron a lo largo de la década de los 90 (ver Alba-Tercedor et al., 1992; Alba-Tercedor \& Prat, 1992; Alba-Tercedor \& Pujante, 2000). La generalidad de estos trabajos, como corresponde a su carácter de pioneros en la prospección de las áreas geográficas estudiadas, se centra en aspectos descriptivos de dinámica espacio-temporal y, con frecuencia, de las relaciones faunacalidad fisico-química del agua. Algunos de los trabajos llevados a cabo en esta vertiente mediterránea han realizado esenciales contribuciones a la mejora del conocimiento taxonómico, corológico y autoecológico de grupos concretos del bentos fluvial (ver listas faunísticas publicadas por la Asociación española de Limnología). Casi paralelamente, surgió el interés por la vertiente aplicada de la macrofauna fluvial, como herramienta para la evaluación de la calidad biológica. Tales estudios de evaluación se han fundamentado, en su mayoría, en la propuesta anglosajona BMWP (Biological Monitoring Working Party) del National Water Council (1981), cuya aplicación a los ríos peninsulares se ha llevado a cabo tras una adaptación (IBMWP: Alba-Tercedor \& Sánchez-Ortega, 1988; Alba-Tercedor, 1996). No obstante, tal adaptación en buena medida debería considerarse como propuesta abierta, debido a la todavía limitada información taxonómica y ecológica sobre la macrofauna fluvial mediterránea (Aguiar et al., 2002). Incluso en regiones con faunas más estudiadas, el actual conocimiento sobre los procesos estructuradores de las comunidades lóticas y de los mecanismos que las alteran en situaciones de estrés es todavía fragmentario (Hildrew, 1992), y su mejora es un objetivo deseable para conseguir una base científica firme en su utilización para la evaluación de la calidad ecológica fluvial (Wright, 2000).

Existe amplio consenso a cerca de que un uso racional, al menos, de los macroinvertebrados como indicadores biológicos, puede ser llevado a cabo con un conocimiento previo de sus patrones de distribución y de las circunstancias biogeográficas y respuestas a los factores de estrés más importantes que los determinan (Plafkin et al., 1989; Rosenberg \& Resh, 1993; 
Marchant et al., 1999; Wright et al., 2000). Tales aspectos, sin duda, estarán muy condicionados por el marco climático, geológico e histórico de cada región en particular. Este conocimiento previo debería permitir el ajuste de dichos índices a las peculiaridades faunísticas y ecológicas de cada región diferenciada y sus gradientes ambientales (Chessman \& McEvoy, 1998, en Griffith et al., 2001), redundando en una mayor precisión de la evaluación. Precisamente, la reciente Directiva Marco del Agua (DMA) (D.O.C.E., 2000) se ha hecho eco de esta necesidad y ha dispuesto que la evaluación de la calidad ecológica de los sistemas acuáticos de la Unión Europea se haga contemplando sus peculiaridades estructurales y dinámicas. Sin embargo, la compilación y sistematización de tal información a partir de estudios realizados a pequeña escala puede resultar ardua y equívoca, no sólo por su escasez, también porque la documentación de la tolerancia ambiental de los taxones no sea el principal objetivo del estudio (Kay et al., 2001). Una solución a estos problemas puede surgir por la vía del desarrollo de trabajos de investigación que se planteen éste como objetivo principal, asegurando una metodología común y ajustada a lo que se pretende, y abarcando amplias áreas geográficas para asegurar la concurrencia de todo el rango de variación de condiciones ambientales en la región. Este es el ánimo que ha guiado el presente trabajo, que, por primera vez en la región del Mediterráneo, aborda un estudio de las comunidades macrobentónicas a gran escala geográfica, sobre 12 cuencas fluviales localizadas de norte a sur de la Península Ibérica e Islas Baleares. Los objetivos específicos del trabajo son: 1) examinar los patrones de distribución de los macroinvertebrados al nivel taxonómico de familia, 2) identificar las variables físicoquímicas fluviales generadoras de los principales gradientes ambientales potenciales condicionantes de estos patrones de distribución, 3) analizar la respuesta que frente a estos gradientes ambientales tienen determinadas familias a las que se les asigna un determinado valor indicador en distintos índices bióticos, y 4) ana- lizar las relaciones de la riqueza de familias con los gradientes ambientales obtenidos y con determinadas características fisiográficas del río y su cuenca.

\section{ÁREA DE ESTUDIO}

Se han estudiado 12 cuencas fluviales, $10 \mathrm{de}$ éstas se encuentran distribuidas de norte a sur a lo largo de la vertiente mediterránea de la Península Ibérica y otras 2 localizadas en la isla de Mallorca (Baleares).

El conjunto de estas cuencas abarca un amplio rango de condiciones del hábitat fluvial consecuencia de la alta diversidad de matices climáticos, de la heterogeneidad topográfica, geológica, de usos del suelo y de la red fluvial en la región. Se encuentran representadas áreas con clima húmedo, cuya precipitación media anual supera los $800 \mathrm{~mm}$, pasando por condiciones climáticas de transición (subhúmedas-secas) con precipitación media anual entre los 300-800 mm, hasta zonas semiáridas-áridas con precipitaciones inferiores a los $300 \mathrm{~mm}$ que son particularmente frecuentes en el extremo sureste de la Península (Capel-Molina, 2000). Esta diversidad climática junto con la gran heterogeneidad geomorfológica y amplio rango altitudinal de las cuencas estudiadas, hace que se encuentren representados muy diversos tipos hidrológicos y geomorfológicos de cauces fluviales: desde ríos con cabeceras en alta montaña (e.g., sobre los 2000 m s.n.m. en Sierra Nevada) sobre materiales predominantemente silíceos y con cierta influencia nival en su régimen; ríos con cabeceras en sistemas montañosos predominantemente calizos de media altitud (e.g., Sierras de los sistemas Bético e Ibérico y estribaciones de Pirineos) con tramos medios y bajos relativamente caudalosos; ríos asociados a sistemas kársticos en caliza o karst en yesos (e.g., Río de Aguas en Almería); torrentes temporales sobre materiales calizos muy permeables (e.g., torrentes de la Sierra de Alfabia en Mallorca); hasta ramblas con alto contenido salino, permanentes o temporales, sobre materiales margosos generalmente próximas al mar, que 
son especialmente frecuentes en el sureste peninsular (e.g., cuenca del Río Segura). Todas las cuencas estudiadas generalmente presentan un considerable grado de actividad antrópica, característica de toda la cuenca del Mediterráneo. La actividad dominante suele ser la agricultura, aunque suelen ser frecuentes e intensas otro tipo de actividades (industriales y servicios) especialmente en tramos medios y bajos de los ríos catalanes y en el Río Segura. No obstante se hallan también representados sectores de redes fluviales, normalmente cabeceras, en áreas bajo algún estatus de protección del medio natural (e.g., Parque de Sant Llorenç del Munt, en la cuenca del Río Llobregat; Parque Natural de Sierra Nevada, cuencas altas de los ríos Guadalfeo y Adra; Paraje Natural del Karst en yesos de Sorbas, cuenca del Río Aguas).

Una descripción detallada de la localización y características fisiográficas de las cuencas estudiadas se presenta en Robles et al. (este volumen).

\section{MATERIAL Y MÉTODOS}

\section{Macroinvertebrados}

En el conjunto de las 12 cuencas se seleccionaron 157 localidades de estudio. La selección se realizó con un criterio de representatividad, tratando de abarcar los gradientes ambientales más característicos de cada cuenca, criterio que forzosamente fue modulado por las condiciones de accesibilidad a los cauces. Estas localidades fueron muestreadas, en general, con una periodicidad trimestral entre abril de 1999 y febrero de 2001, que en algunas cuencas estuvo condicionada por la temporalidad de los ríos. En cada localidad y fecha se tomó una muestra de la comunidad de macroinvertebrados con una red de $250 \mu \mathrm{m}$ de poro de malla, integrando los distintos hábitats fluviales diferenciados de visu en un tramo de $100 \mathrm{~m}$ de longitud. Los macroinvertebrados fueron identificados hasta nivel taxonómico de familia (excepto oligoquetos, ostrácodos e hidrácaros) (Tachet et al., 2000). Una vez concluida la fase de identificación, sobre una submuestra de 200 individuos, a cada taxon, en cada muestra, le fue asignado un rango de abundancia: 1 (1-3 individuos), 2 (4-10 individuos), 3 (11-100 individuos) y 4 (más de 100 individuos). Para cada muestra se calcularon las riquezas del total de familias, riqueza de familias EPT (efemerópteros, plecópteros y tricópteros) y $\mathrm{OCH}$ (odonatos, coleópteros y heterópteros), y valores de los índices bióticos IBMWP e IASPT (AlbaTercedor, 1996). Una descripción detallada sobre el protocolo de muestreo de campo y técnicas de laboratorio se expone en Jáimez-Cuéllar et al. (este volumen).

\section{Variables abióticas}

En cada localidad y por cada fecha de muestreo se midieron in situ temperatura, $\mathrm{pH}$ y conductividad del agua, mediante sensores portátiles, y el caudal, que se estimó midiendo la corriente con un correntímetro en una sección transversal del río de superficie conocida. Los análisis de concentración en agua de amonio, nitritos, nitratos, y fosfatos se llevaron a cabo in situ mediante equipos portátiles o en laboratorio. Para cada localidad, se determinó su altitud, área de cuenca drenada y orden del tramo del río mediante mapas topográficos de escala 1:200.000. Un informe pormenorizado de las metodologías de campo y técnicas analíticas de laboratorio se presenta en Toro et al. (este volumen) y JáimezCuéllar et al. (este volumen).

\section{Análisis estadísticos}

Para explorar las principales tendencias de variación de las familias de macroinvertebrados y su relación con las variables fisico-químicas del agua estudiadas se han aplicado dos tipos de análisis de ordenación: análisis de correspondencias (AC), técnica de análisis indirecto de gradiente, y análisis canónico de correspondencias (ACC), técnica de análisis directo de gradiente. Ambos tipos de análisis fueron llevados a cabo mediante el paquete estadístico CANO$\mathrm{CO}$ en versión 4 para Windows (ter Braak \& Smilauer, 1998). En el análisis indirecto (AC) 
las familias de invertebrados se ordenaron sin ser condicionadas por variable ambiental alguna, mientras que en el análisis directo de gradiente (ACC) la matriz de variables dependientes (familias de macroinvertebrados) se ordenó siendo condicionada (constreñida) por la matriz de variables independientes (variables físicoquímicas del agua en nuestro caso). De acuerdo con ter Braak (1986) si las ordenaciones de "especies" resultantes de los análisis directo e indirecto no resultan fundamentalmente diferentes, se puede considerar que ninguna variable ambiental importante ha sido pasada por alto. La correspondencia entre ordenaciones de las muestras (AC vs. ACC) se estudió mediante el análisis de correlación de Pearson de los valores de las muestras en los correspondientes ejes canónicos.

Ambos tipos de análisis (AC y ACC) se llevaron a cabo con una matriz de rango de abundancia de las familias, tras ser suprimidas aquellas que se recolectaron en 5 o menos muestras (20\% de las familias censadas). La matriz de variables ambientales estuvo compuesta por las variables fisico-químicas medidas en el agua del río ( $\mathrm{pH}$, conductividad, temperatura, caudal, amonio, N-nitritos, N-nitratos y P-fosfatos), que previamente fueron transformadas logarítmicamente $(\ln \mathrm{x}+1)$ excepto $\mathrm{pH}$. Se estudió además su colinearidad mediante análisis de correlación de Pearson. Sólo las concentraciones de P-fosfatos y amonio presentaron valor de correlación superior a $0.5(p<0.05)$. No obstante, para el análisis ACC no se eliminó ninguna de las dos variables anteriores ya que sus factores de inflación no superaron el valor de 20 (ter Braak \& Smilauer, 1998).

En un análisis ACC previo se detectó que 32 muestras presentaron valores extremos para determinadas variables ambientales (amonio, $\mathrm{N}$ nitritos y P-fosfatos). Estas muestras fueron eliminadas en un segundo análisis definitivo. Para ambos tipos de análisis, AC y ACC, se escogió para la medida de la distancia entre "especies" la opción del ajuste de Hill, ya que resulta más adecuada cuando se trabaja con amplios gradientes ambientales (ter Braak \& Smilauer,
1998); por lo demás fueron aceptadas las opciones por defecto. La significación estadística del primer eje y de la suma de todos los ejes canónicos para el análisis ACC fue contrastada mediante el test de Monte Carlo usando permutaciones no restringidas (199 permutaciones). Se utilizó el coeficiente de correlación de Spearman para estudiar la relación entre las tres primeras dimensiones canónicas y las tres variables fisiográficas medidas, para una más completa interpretación de tales dimensiones.

Se llevaron a cabo análisis de correlación mediante el test no paramétrico de Spearman para estudiar la relación de riqueza total de familias, riqueza de familias por ordenes de insectos, moluscos y crustáceos, riqueza de familias de grupos EPT (efemerópteros, plecópteros y tricópteros) y $\mathrm{OCH}$ (odonatos, coleópteros y heterópteros) y valores resultantes de los índices bióticos IBMWP e IASPT, con las variables fisico-químicas del agua y fisiográficas de cada localidad (altitud, orden del río y área de cuenca drenada), y con los tres primeros gradientes ambientales (ejes canónicos) extraídos por el análisis ACC. Estos análisis de correlación se repitieron una vez eliminadas de la matriz de datos todas las muestras con claros síntomas de contaminación según los siguientes cortes de concentración de nutrientes: amonio $>0.1 \mathrm{mg} / \mathrm{l} ; \mathrm{N}$-nitrito $>0.05 \mathrm{mg} / \mathrm{l}$; P-fosfato $>0.1 \mathrm{mg} / \mathrm{l}$. El objetivo de esta segunda tanda de análisis de correlación fue disociar la mineralización (conductividad) natural de la debida a solutos relacionados con eventos de contaminación, para poder interpretar con mayor certidumbre la correlación entre mineralización natural del agua y riquezas de familias de macroinvertebrados e índices bióticos.

\section{RESULTADOS}

\section{Composición taxonómica}

Ciento veintisiete taxones de macroinvertebrados, identificados mayoritariamente hasta familia, fueron censados en el total de las 12 cuencas 
fluviales estudiadas (Anexo 1). El dominio taxonómico correspondió a los insectos con 101 familias censadas (79\%), y dentro de éstos, dípteros (20 familias) y tricópteros (15 familias) fueron los ordenes mejor representados. Los grupos con mayor frecuencia de aparición fueron Chironomidae (presente en el $96 \%$ de las muestras), Baetidae ( $89 \%$ de las muestras) y Oligochaeta (70 \% de las muestras). Tan sólo 4 familias más aparecieron en más del $50 \%$ de las muestras: Caenidae, Dytiscidae, Hydropsychidae y Simuliidae. Un $52 \%$ de los taxones (66 taxones) fueron censados en menos del $10 \%$ de las muestras. Veinticinco familias aparecieron muy esporádicamente (en 5 o menos muestras), las más raras fueron: Corduliidae, Ecnomidae, Prosopistomatidae y Hebridae. El número medio de taxones por muestra fue de 18 , mientras que el máximo número de taxones por muestra fue de 46 que se censaron en 2 localidades de la cuenca del Río Segura (Murcia) y una localidad del Río Almanzora (Almería).

\section{Análisis canónicos}

Los autovalores resultantes para las 4 primeras dimensiones canónicas extraídas por AC y ACC (Tabla 1) son relativamente bajos como era de esperar del amplio número de taxones y muestras que constituyen la matriz de datos analizada, además de la considerable heterogeneidad de estas. Esto se refleja también en los relativamente bajos porcentajes de varianza explicados por cada eje (Tabla 1). No obstante, probablemente resulta más interesante destacar en el presente contexto el contraste de autovalores y porcentaje de varianza explicada entre los ejes de AC y ACC. El autovalor y porcentaje de varianza explicado por la primera dimensión ACC representan un alto porcentaje, alrededor de un $75 \%$, de la misma dimensión AC, indicando que las variables ambientales relacionadas con este gradiente lo explican muy satisfactoriamente. Las restantes dimensiones canónicas ACC no llegan a representar más del $40 \%$ (autovalores o porcentaje de varianza

Tabla 1. Resumen de los resultados del análisis de correspondencias (AC) y del análisis canónico de correspondencias (ACC). Autovalores y porcentaje de varianza acumulada de la matriz de taxones explicado por las cuatro dimensiones AC y ACC. Coeficientes de correlación entre taxones-variables ambientales para el ACC. Los valores entre paréntesis indican el porcentaje que representan las dimensión ACC de las correspondientes AC. La significación estadística (test de Monte Carlo) se ha calculado para el primer eje del ACC y para todos los ejes combinados. Summary of results from correspondence analysis (CA) and canonical correspondence analysis (CCA). Eigenvalues and percentage of variance explained of the taxa by canonical dimensions in the CA and CCA. Canonical correlation coefficients between taxa and environmental variables for CCA are also shown. Values between parentheses are percentages represented by CCA dimensions of the corresponding CA. Statistical significance (Monte Carlo test) has been calculated for the first and all dimensions pooled of CCA.

\begin{tabular}{|c|c|c|c|c|}
\hline & EJE1 & EJE2 & EJE3 & EJE4 \\
\hline \multicolumn{5}{|l|}{ Autovalores } \\
\hline $\mathrm{AC}$ & 0.28 & 0.17 & 0.16 & 0.12 \\
\hline $\mathrm{ACC}$ & $0.21(75 \%)$ & $0.06(35 \%)$ & $0.05(31 \%)$ & $0.04(33 \%)$ \\
\hline \multicolumn{5}{|c|}{$\%$ de varianza acumulado de los taxones } \\
\hline $\mathrm{AC}$ & 7.0 & 11.3 & 15.3 & 18.3 \\
\hline $\mathrm{ACC}$ & $5.2(74 \%)$ & $6.8(37 \%)$ & $8.0(30 \%)$ & $9.1(37 \%)$ \\
\hline \multicolumn{5}{|c|}{ Coeficientes de correlación canónica entre taxones - ambiente } \\
\hline ACC & 0.88 & 0.66 & 0.59 & 0.67 \\
\hline \multicolumn{5}{|l|}{ Test de Monte Carlo } \\
\hline Eje 1 del ACC & $\mathrm{F}=40.58$ & $\mathrm{p}<0.001$ & & \\
\hline Todos los ejes del ACC & $\mathrm{F}=10.89$ & $\mathrm{p}<0.001$ & & \\
\hline
\end{tabular}


Tabla 2. Coeficientes de correlación de Pearson entre los cuatro primeros ejes del ACC y las variables ambientales incluidas en el análisis. Nivel de significación: $* \mathrm{p}<0.001$. Pearson correlation coefficients between axes of CCA and the environmental variables included in the analysis. Level of significance: ${ }^{*} p<0.001$.

EJE 1 EJE 2 EJE 3 EJE 4

\begin{tabular}{lclll}
\hline AMONIO & 0.10 & $0.19^{*}$ & $0.58^{*}$ & 0.06 \\
N-NITRITOS & 0.00 & $0.20^{*}$ & $0.20^{*}$ & 0.09 \\
N-NITRATOS & 0.03 & 0.12 & 0.00 & $0.59^{*}$ \\
P-FOSFATOS & 0.12 & $0.19^{*}$ & $0.43^{*}$ & 0.11 \\
CAUDAL & $-0.41^{*}$ & $0.58^{*}$ & -0.05 & 0.05 \\
pH & $-0.18^{*}$ & 0.09 & -0.07 & 0.06 \\
TEMPERATURA & $0.49^{*}$ & $0.16^{*}$ & $-0.18^{*}$ & 0.09 \\
CONDUCTIVIDAD & $0.79^{*}$ & $0.15^{*}$ & $0.15^{*}$ & $-0.13^{*}$ \\
\hline
\end{tabular}

explicada) de las equivalentes $\mathrm{AC}$, por lo que las variables ambientales seleccionadas no explican tan satisfactoriamente estas dimensiones como la primera. Cuando se contrasta la ordenación de muestras proporcionada por ambos tipos de análisis mediante la correlación de los valores que obtienen estas en los respectivos ejes $\mathrm{AC}$ frente a ACC, los coeficientes resultaron estadísticamente significativos para las cuatro dimensiones $(\mathrm{p}<0.001)$. No obstante, para las tres primeras dimensiones los valores de correlación resultaron muy altos (primera, $\mathrm{R}=0.99$; segunda, $\mathrm{R}=0.88$; tercera, $\mathrm{R}=0.85$ ) en comparación con la cuarta dimensión $(\mathrm{R}=0.46)$. Tales resultados subrayan la mayor consistencia de las tres primeras dimensiones, en particular la primera, y de las variables ambientales más correlacionadas con estas, y sugieren la escasa conveniencia de interpretar la cuarta dimensión a la luz del limitado número de variables ambientales barajadas por el ACC.

La primera dimensión ACC presentó un alto valor de correlación positiva con la variable conductividad eléctrica, y correlaciones algo menores con temperatura y caudal (Tabla 2). Además, este primer gradiente se correlacionó negativamente con la altitud de las localidades de estudio $\left(\mathrm{R}_{\mathrm{S}}=-0.58, \mathrm{p}<0.001\right)$, lo que permite interpretarlo como un gradiente fluvial longitudinal fundamentalmente de aumento de la salinidad-termicidad. Las cuencas con cabeceras a mayor altitud (e.g., Guadalfeo, Llobregat, Adra) y baja mineralización, presentan los valores más negativos en esta primera dimensión. En el extremo opuesto, con los valores más positivos, se posicionan un gran número de muestras de las cuencas en clima más xérico, a baja altitud y aguas fuertemente mineralizadas del sureste peninsular (cuencas del Aguas y partes bajas del Segura y Almanzora) (Fig. 1). Dentro de cada cuenca se advierte un gradiente espacial longitudinal cabecera-desembocadura que, lógicamente, resulta más extenso para aquellas cuencas con cabeceras montanas y tramos bajos que drenan materiales sedimentarios de naturaleza margosa (Ríos Segura y Almanzora) (Fig. 1). Todas las familias censadas de plecópteros y la mayoría de efemerópteros y tricópteros se decantan hacia las posiciones más negativas de esta dimensión, mientras que en el extremo opuesto se sitúan los dípteros de la familia Ephydridae y moluscos de la familia Thiaridae, y en posiciones menos extremas, los heterópteros Pleidae y Naucoridae, y los coleópteros Noteridae (Fig. 2). La mayoría de las familias se sitúan, no obstante, en posiciones intermedias a lo largo de esta primera dimensión.

La segunda dimensión canónica se correlacionó positivamente sobre todo con el caudal (Tabla 2). La variable fisiográfica que se correlacionó más estrechamente con el eje 2 fue el área de cuenca $(\mathrm{R}=0.67, \mathrm{p}<0.001)$. En el extremo positivo de esta dimensión se sitúan localidades de tramos bajos de las cuencas más extensas con caudales que superan $1 \mathrm{~m}^{3} / \mathrm{s}$ de los ríos Jucar, Turia o Llobregat (Fig. 1). En el extremo negativo de esta dimensión aparecen localidades con caudales inferiores a los 50 1/s con diferente localización en sus respectivas cuencas: cabeceras de las cuencas con mayor gradiente altitudinal; numerosas localidades de cuencas pequeñas sobre sustratos muy permeables, como los torrentes de la isla de Mallorca; o localidades de tramos mediosbajos de las cuencas del sureste con carácter temporal o intermitente (Fig. 1). Las familias más estrechamente asociadas con caudales altos son los efemerópteros Polymitarcidae, Oligoneuriidae y Potamantidae, y los crustáceos de la familia Atyidae (Fig. 2). En el extremo negativo de esta 

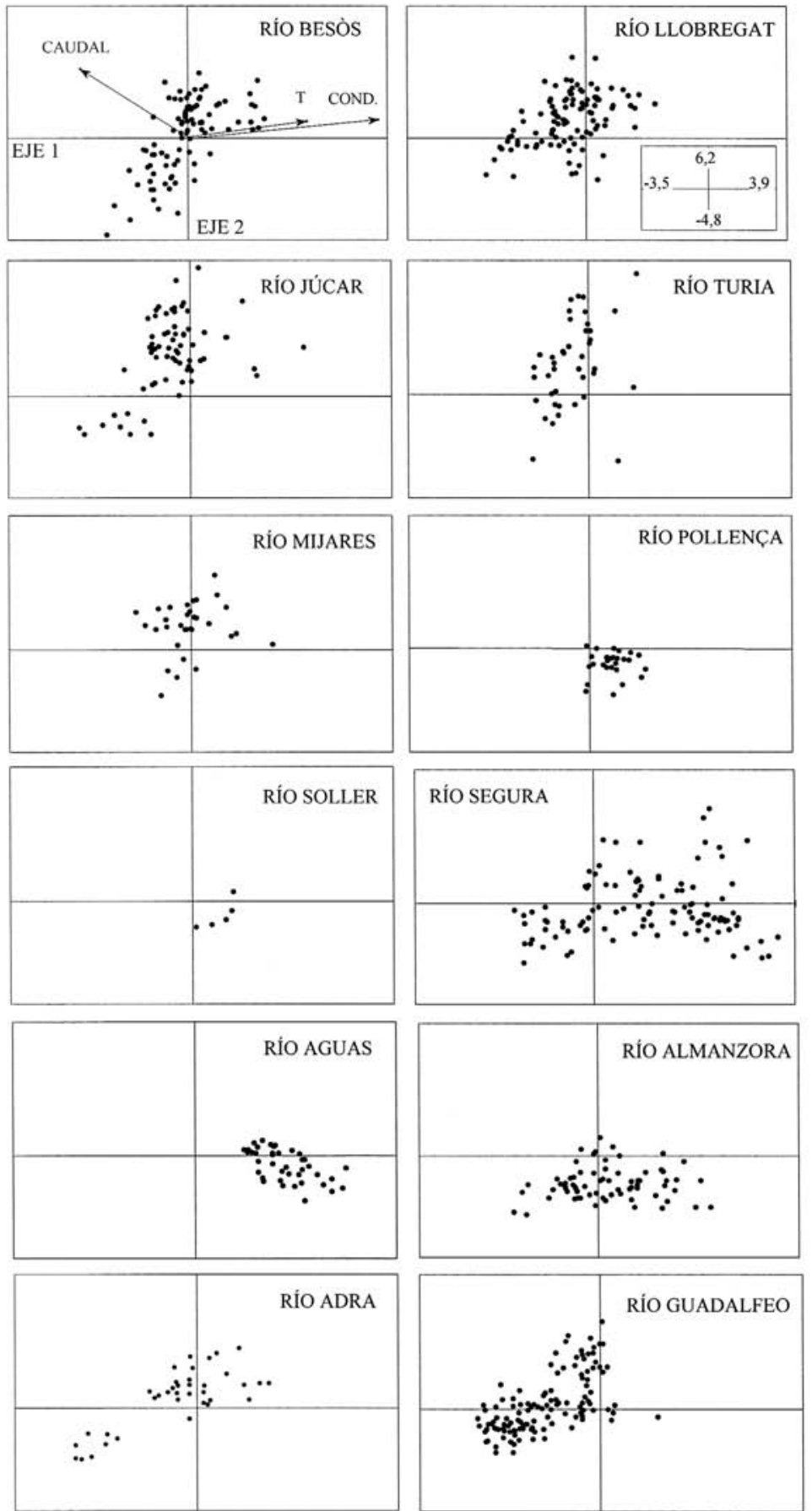

Figura 1. Diagrama de ordenación resultante del análisis canónico de correspondencias (ACC) (ejes 1 y 2) en el que se representan las muestras (localidades de estudio en distintas fechas) por cuencas. Se indican las variables ambientales más correlacionadas con las dimensiones canónicas mediante vectores. La escala para todos los gráficos se indica en la gráfica del Río Llobregat. Las puntuaciones de los vectores ambientales se multiplicaron por cuatro para mayor claridad gráfica. Ordination plot of samples pooled by basin (axes 1 and 2) from the canonical correspondence analysis (CCA). Vectors indicate the environmental variables most correlated with the canonical dimensions. The corresponding scale is shown in the plot of the River Llobregat (Barcelona). The scores of environmental vectors were multiplied by four for graphical clarity. 

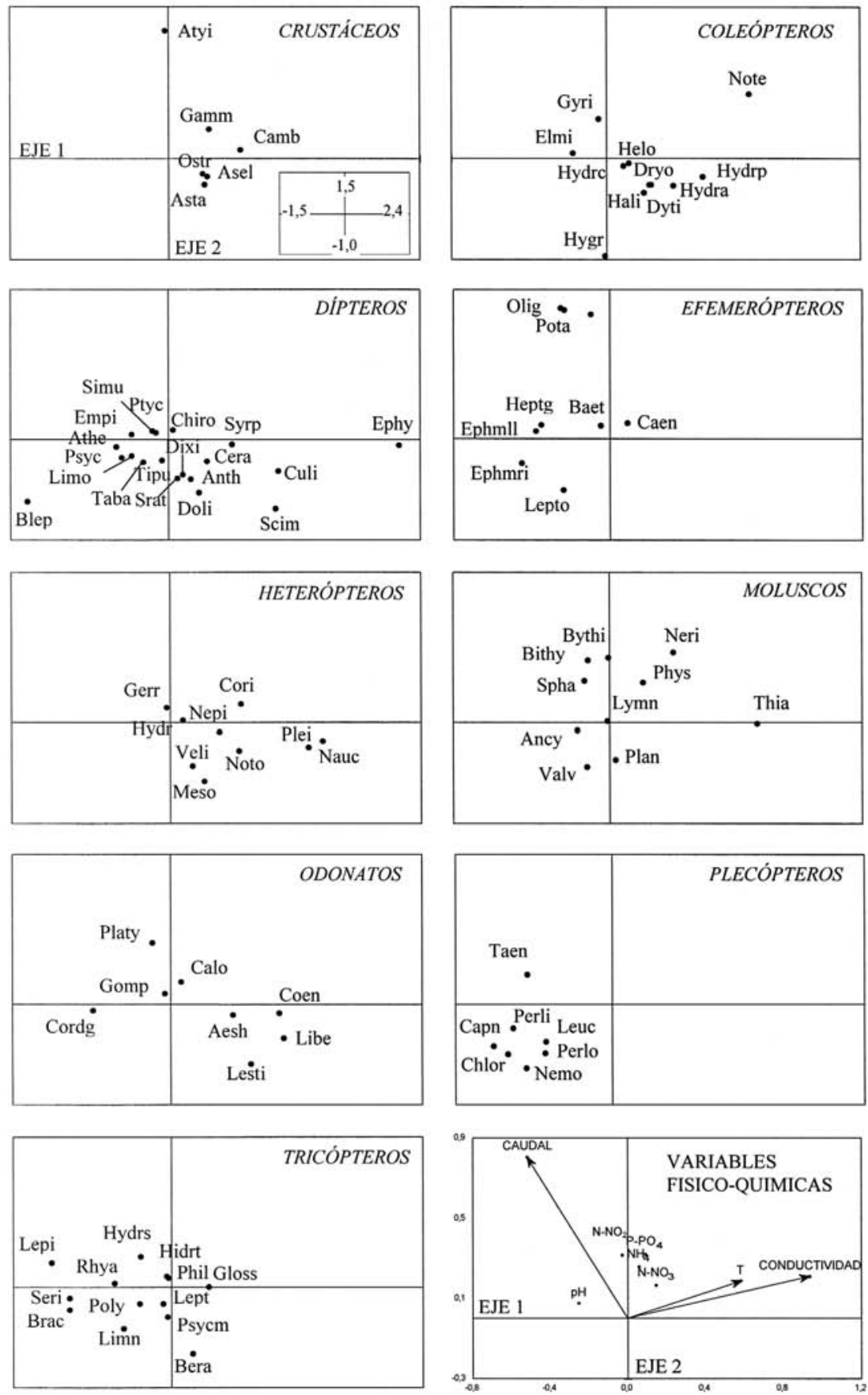

Figura 2. Diagrama de ordenación ACC (ejes 1 y 2) en el que se representan las familias agrupadas por ordenes de insectos, moluscos y crustáceos. Se representan también mediante vectores las variables ambientales más correlacionadas con las dimensiones del ACC. Las familias aparecen designadas por abreviaturas cuya expresión completa se presenta en el Anexo 1. La escala para todos los gráficos de los taxones se indica en la gráfica de los crustáceos. Ordination plot CCA (axes 1 and 2) showing the families grouped by orders of insects, and mollusc and crustaceans. Vectors indicate the environmental variables most correlated with the canonical dimensions. Family codes are explained in Annex 1. The corresponding scale is shown in the plot of crustaceans. 
dimensión se sitúan varias familias de plecópteros y algunos tricópteros, características de cabeceras, y por otra parte numerosas familias de dípteros, odonatos, heterópteros y coleópteros, características de tramos muy mineralizados y térmicos, con muy bajo caudal o caudal nulo que conduce a un predominio de ambientes leníticos (Fig. 2).

La tercera dimensión canónica se relaciona principalmente con eventos de contaminación del agua, a juzgar por las importantes correlaciones con amonio y P-fosfato (Tabla 2). Este gradiente ambiental segrega en el extremo positivo numerosas localidades de tramos medios y bajos de las dos cuencas catalanas que drenan áreas de alta densidad de población y presentan usos agrícolas e industriales intensivos. No obstante, casi todas las cuencas presentan alguna localidad y/o muestra claramente decantada hacia este extremo positivo (Fig. 3). En este polo se ordenan pocas familias, la más extrema resultó Asellidae, y en posiciones más centradas se ordenan Syrphidae, Chironomidae y Physidae, y también algunas familias de plecópteros, especialmente Taeniopterigidae (Fig. 4). Con la posición más negativa en esta dimensión se ordenan Atyidae, Ptychopteridae, Oligoneuridae y Polymitarcidae (Fig. 4).

\section{Riqueza de familias e índices bióticos}

La riqueza total de familias sólo presentó correlación negativa con significación estadística con la tercera dimensión canónica (contaminación) y con los dos nutrientes que explican esta dimensión (Tabla 3). Ninguna de las tres variables fisiográficas mostró correlación significativa con esta variable. Las riquezas EPT y OCH presentaron patrones de correlación contrapuestos con la primera dimensión canónica (salinidad-termicidad), negativa y positiva respectivamente (Tabla 3), aunque ambos grupos convergen en su correlación negativa con la dimensión contaminación (Tabla 3). Los ordenes componentes de ambos grupos presentaron bastante congruencia intragrupo en las tendencias y magnitud de las correlaciones con las variables y gradientes ambientales anteriormente mencionados (Tabla 3). No obstante, es des- tacable el bajo valor de la correlación negativa de la riqueza de plecópteros con la dimensión canónica de contaminación, que contrasta con la del resto de órdenes. Cuando se suprimieron del análisis de correlación aquellas muestras con aguas más contaminadas, como era de esperar, disminuyó la fuerza de la correlación negativa de la riqueza de órdenes con esta dimensión , aunque cabe destacar que aumentó la magnitud de las correlaciones positivas de las riquezas de los órdenes OCH con la dimensión canónica asociada a salinidad-termicidad (Tabla 3 ). Las riquezas de dípteros y plecópteros presentaron las correlaciones (negativas) más altas con la dimensión canónica asociada a caudal. Estos valores de correlación, aunque disminuyeron al suprimir del análisis las muestras más contaminadas, se mantuvieron altamente significativos. La riqueza de crustáceos mostró correlaciones positivas de mayor magnitud con la dimensión salinidad-termicidad, especialmente con la variable conductividad asociada a esta dimensión; tales correlaciones se acentuaron tras extraer del análisis las muestras más contaminadas (Tabla 3 ).

La puntuación proporcionada por el índice IBMWP presentó fuertes correlaciones negativas con la dimensión contaminación y las concentraciones de nutrientes asociados a ésta (Tabla 3), mientras que los valores de las clases de calidad de este mismo índice presentaron valores de correlación semejantes a los de la puntuación del índice, aunque de signo contrario, con las variables ambientales mencionadas. Son destacables los valores relativamente altos de correlación de las dos mencionadas variables indicadoras de calidad con las dimensiones salinidad-termicidad (principalmente con la variable conductividad) y caudal. Los valores de correlación (negativa) del índice IASPT con la dimensión salinidad-termicidad, además de con las variables conductividad y altitud, fueron aún mayores, mientras que los valores absolutos de correlación con la dimensión contaminación, y especialmente con las concentraciones de nutrientes asociados a ésta dimensión, resultaron considerablemente más bajos. 


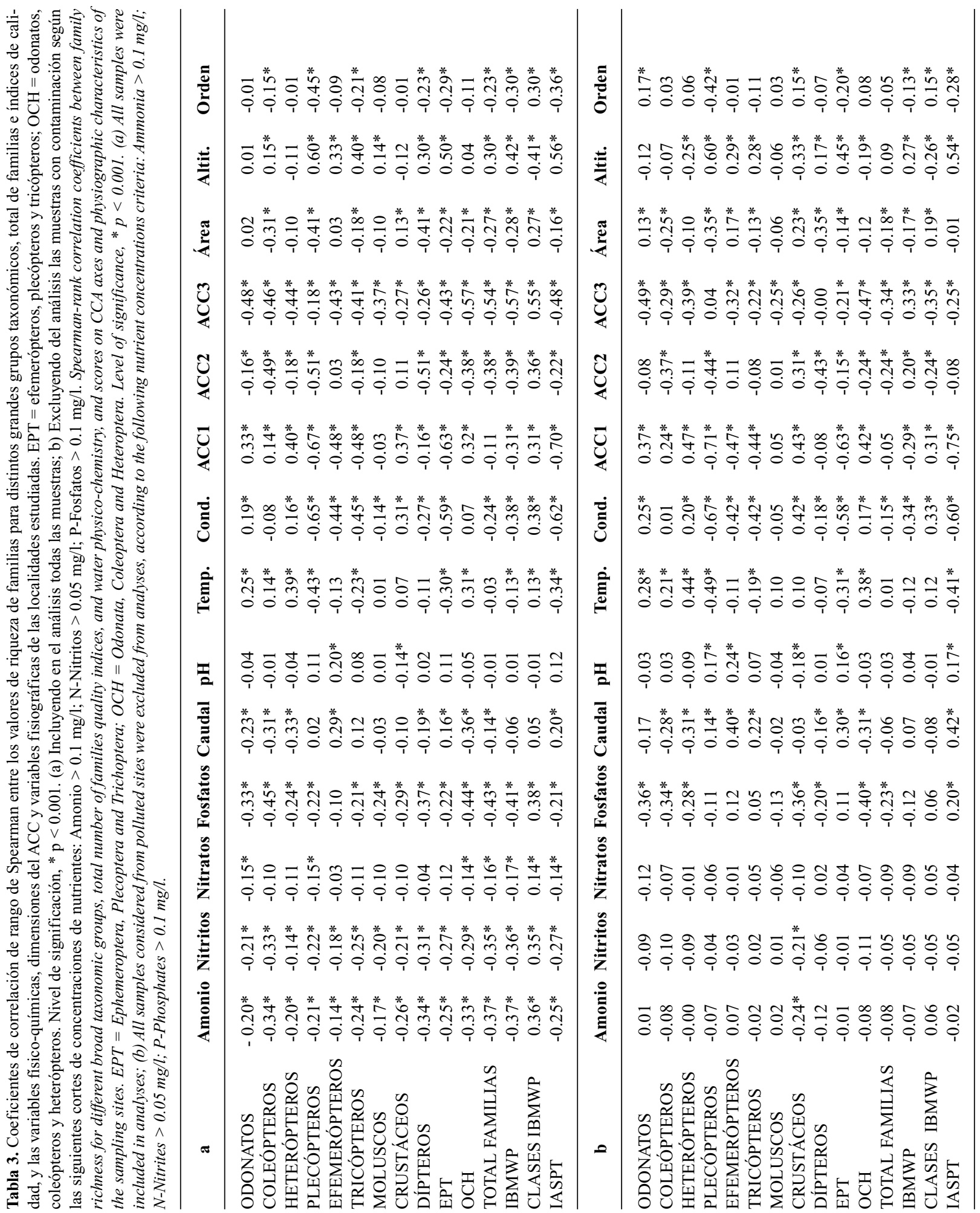



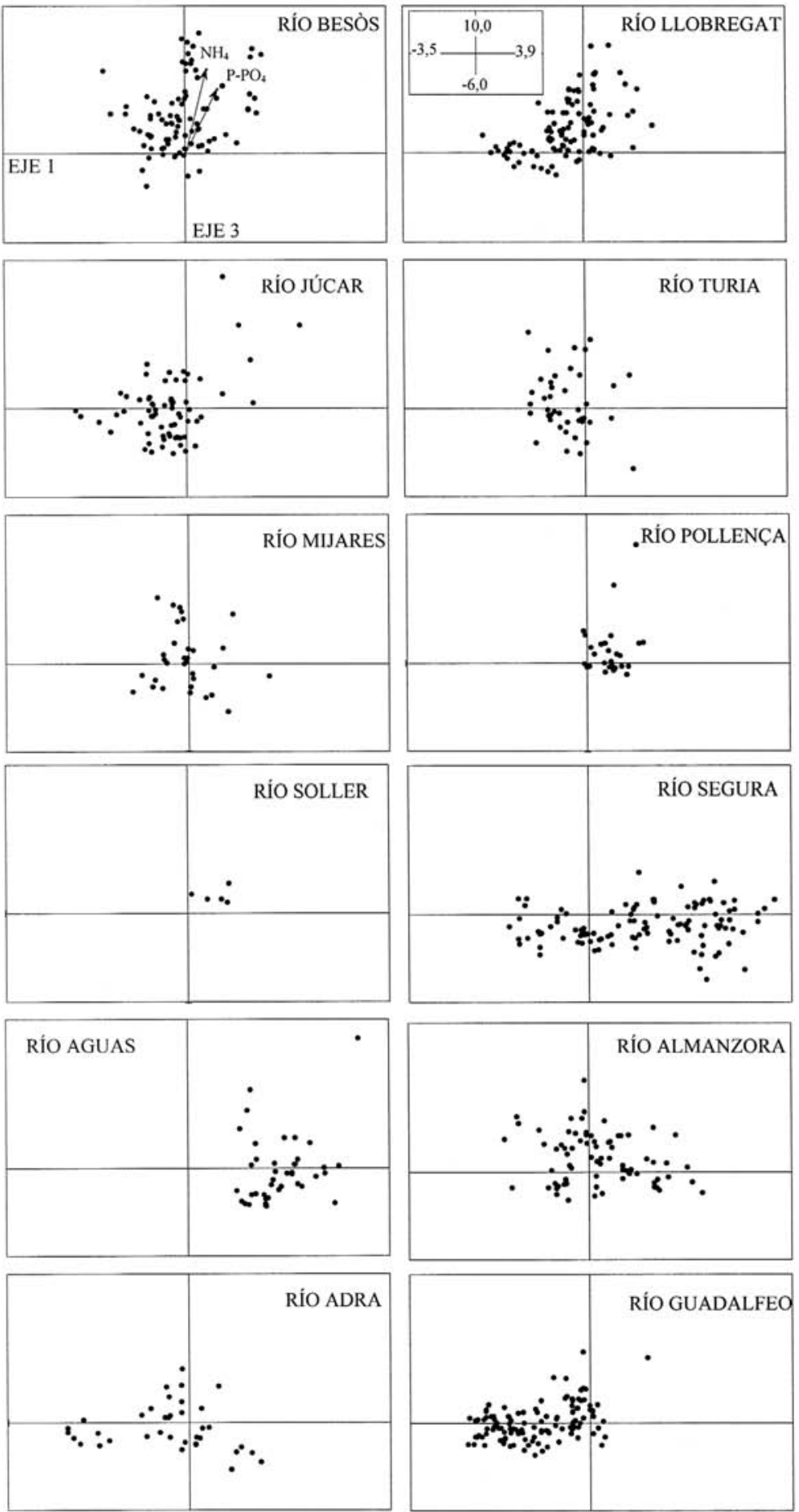

Figura 3. Representación de las mismas variables que para la figura 1 aunque en el espacio determinado por las dimensiones canónicas 1 y 3 . Las coordenadas de los vectores ambientales se multiplicaron por diez para mayor claridad gráfica. Plot showing the same variables as for figure 1 but in the plane defined by the canonical dimensions 1 and 3. The scores of environmental vectors were multiplied by ten for graphical clarity. 


\section{DISCUSIÓN}

Las características fisico-químicas del medio acuático suelen ejercer una importante influencia sobre la distribución de los macroinvertebrados fluviales (e.g., Williams \& Feltmate, 1992; Lampert \& Sommer, 1997; Giller \& Malmqvist, 1998). La general sensibilidad de éstos a variables como velocidad de la corriente, temperatura, concentraciones de oxígeno y materia orgánica, y salinidad, en parte, los ha convertido en una valiosa herramienta para el biomonitoreo (ver Rosenberg \& Resh, 1993).

En el presente estudio, el relativamente bajo porcentaje de varianza explicado por las tres primeras dimensiones del análisis AC (aproximadamente $15 \%$ del total) es, muy probablemente, un reflejo de la gran magnitud de las escalas espacio-temporales que incluye la matriz de familias analizada, junto con el gran número de muestras y taxones implicados, y también, quizás, de la escasa resolución taxonómica de su identificación. Por otra parte, las 8 variables físico-químicas seleccionadas para el análisis ACC explican poco más del $8 \%$ de la varianza total de la matriz de familias de macroinvertebrados. No obstante, en estudios realizados en otras regiones bajo clima mediterráneo, abarcando una menor escala espaciotemporal y resolviendo hasta nivel de especie en la mayoría de las identificaciones, los patrones de variación de los macroinvertebrados fluviales también resultaron pobremente explicados (Cortes et al., 1998), incluso cuando se disponía de nutridas matrices de variables ambientales como las utilizadas por Aguiar et al. (2002) en una cuenca del sur de Portugal. El relativamente alto régimen de perturbación, natural y/o antrópica, al que suelen estar sometidos los sistemas fluviales mediterráneos ha sido con frecuencia argüido como responsable del predominio de taxones altamente ubiquistas y, por tanto, escasamente condicionados por las variables ambientales típicamente consideradas como importantes en estos sistemas (Gasith \& Resh, 1999; Aguiar et al., 2002). Kay et al. (2001) añaden que, especialmente, la intermitencia y temporalidad característica de los ríos en climas con cierto grado de aridez, suelen acentuar sobremanera la irregularidad en la distribución espacio-temporal de los macroinvertebrados, lo que puede acrecentar el ruido en la búsqueda de relaciones taxones-variables ambientales. Según estos autores, en estos ríos abundan los hábitats efímeros y esporádicos específicos de especies con altas capacidades de colonización y emigración que conducen a una fuerte irregularidad de ocurrencia. De hecho, en el presente estudio el $40 \%$ de las localidades se mostraron intermitentes o temporales y, aunque la mayor parte de las familias mostraron una extensa distribución geográfica (presentes en la mayoría de cuencas estudiadas), más del $50 \%$ de éstas fueron censadas en menos del $10 \%$ del total de las muestras obtenidas, lo que pone de manifiesto la acentuada irregularidad antes mencionada.

Debemos también considerar que, obviamente, un número tan limitado de variables ambientales, como el utilizado en el presente estudio, por muy esenciales que éstas sean para los macroinvertebrados fluviales, no permitan explicar con muy alta resolución una matriz de familias tan rica y compleja. No obstante, ya es altamente satisfactorio que esta matriz ambiental permita satisfacer algo más de la mitad de la pura varianza (AC) absorbida por las 3 primeras dimensiones canónicas. Planteado en estos términos, podemos afirmar con bastante certidumbre que, al menos, las 6 variables físico-químicas notablemente correlacionadas con estas 3 primeras dimensiones, son altamente relevantes para el conjunto de los macroinvertebrados.

\section{Salinidad-termicidad}

La salinidad y la temperatura son las principales variables que condicionan la distribución de las familias de macroinvertebrados fluviales en nuestra área de estudio. Estas variables generan un gradiente ambiental de aumento de la salinidad-termicidad que se relaciona negativamente con la altitud y el caudal. Un rasgo distintivo de los ríos de zonas áridas es que pueden ser considerablemente salinos, en parte 
como consecuencia de la alta evaporación, pero también porque la escorrentía procedente de la cuenca aporte cloruros $\mathrm{u}$ otras sales bastante solubles desde sedimentos marinos fósiles (Giller \& Malmqvist, 1998). En la región estudiada, mientras que numerosas cabeceras de cuenca drenan materiales poco solubles en un marco climático húmedo, los dos eventos anteriormente mencionados concurren en algunas cuencas, con especial incidencia en áreas próximas al litoral marino del sureste de la Península Ibérica. El caso más extremo en el gradiente de salinidad-termicidad lo representan las "ramblas hipersalinas" de la cuenca baja del Río Segura, sobre un sustrato margoso y en ambiente semiárido que pueden alcanzar valores en el agua de conductividad eléctrica superiores a los $50 \mathrm{mS} / \mathrm{cm}$, y temperaturas máximas superiores a $30{ }^{\circ} \mathrm{C}$ debido al bajo caudal y la ausencia en la ribera de dosel arbóreo. En algunas regiones de Australia, Norte América, África y Oriente Próximo, con frecuencia enmarcadas por condiciones de aridez, a los agentes naturales causantes de salinización de los sistemas acuáticos continentales, se suman causas antrópicas principalmente ligadas a la agricultura, en un proceso que se suele denominar salinización secundaria (Williams, 1987; Collier, 1995; Leland \& Fend, 1998; Shieh et al., 1999; Kay et al., 2001). Sin embargo, en la región estudiada, a pesar del dominante uso agrícola de muchas de las cuencas vertientes, la salinidad no se relaciona significativamente con las variables indicadoras de fertilización antropogénica, ni tampoco con un buen indicador de contaminación difusa por actividades agrícolas como suele ser la concentración de nitrato, por lo que ésta puede ser tenida como una condición sujeta a variabilidad esencialmente natural. Incluso, en algunos sistemas de ramblas saladas de la región de Murcia parece estar registrándose un proceso inverso, consistente en la dulcificación a consecuencia de la puesta en regadío de zonas próximas de la cuenca vertiente (M.L. Suárez y M.R. Vidal-Abarca, datos no publicados).

Este gradiente ambiental salinidad-termicidad se muestra altamente selectivo para un buen número de familias. El orden de macroinvertebrados que parece mostrarse más estenoico hacia el extremo bajo de este gradiente es el de plecópteros, seguido de efemerópteros y tricópteros; la riqueza de familias de los tres órdenes se correlaciona negativamente con esta dimensión. La temperatura se considera el factor ambiental más importante que condiciona la eclosión de los huevos, el desarrollo de las ninfas, y en general, la distribución de las especies de plecópteros, de tal forma que estos sólo aparecen si la temperatura se mantiene por debajo de $25^{\circ} \mathrm{C}$ (Harper, 1979; Stewart \& Stark, 1988). No obstante, nuestros datos demuestran que algunas familias pueden estar presentes por encima de este umbral térmico. Por otra parte, en el estudio de un río mediterráneo, Prenda \& Gallardo (1999) subrayan que para explicar la distribución longitudinal de los plecópteros, a su general psychrofilia (sensu lato), hay que sumar la escasa tolerancia de este grupo a alta mineralización del agua, aunque Thyrrenoleuctra (Leuctridae) parece tolerar altas concentraciones salinas (hasta $8.4 \mathrm{mS} / \mathrm{cm}$ ). En el presente estudio un $25 \%$ de las localidades superan $\operatorname{los} 25^{\circ} \mathrm{C}$, rasgo que se solapa sobremanera con aguas fuertemente mineralizadas, escasa elevación sobre el nivel de mar, muy bajo caudal y casi ausencia de corriente. La tolerancia térmica y osmótica de tricópteros y efemerópteros, como grupos, es más amplia comparada con la de plecópteros, ya que algunos taxones están adaptados para medrar en aguas leníticas, cálidas y/o salobres e incluso athalasohalinas, aunque la mayor parte de las familias pertenecientes a estos grupos suelen distribuirse en aguas dulces, corrientes y relativamente frías (Williams \& Felmate, 1992; Wiggins, 1996; Tachet et al., 2000). La distribución de tricópteros ha sido también relacionada con la magnitud y calidad de la cubierta vegetal riparia (Wiberg-Larsen et al., 2000). En la región estudiada, la calidad y grado de cobertura de la vegetación de ribera resultó bastante dependiente del gradiente salinidad-termicidad (Suárez et al., este volumen) por lo que ambos factores podrían solaparse aquí para determinar la distribución observada para este grupo de familias, y probablemente de otras. 

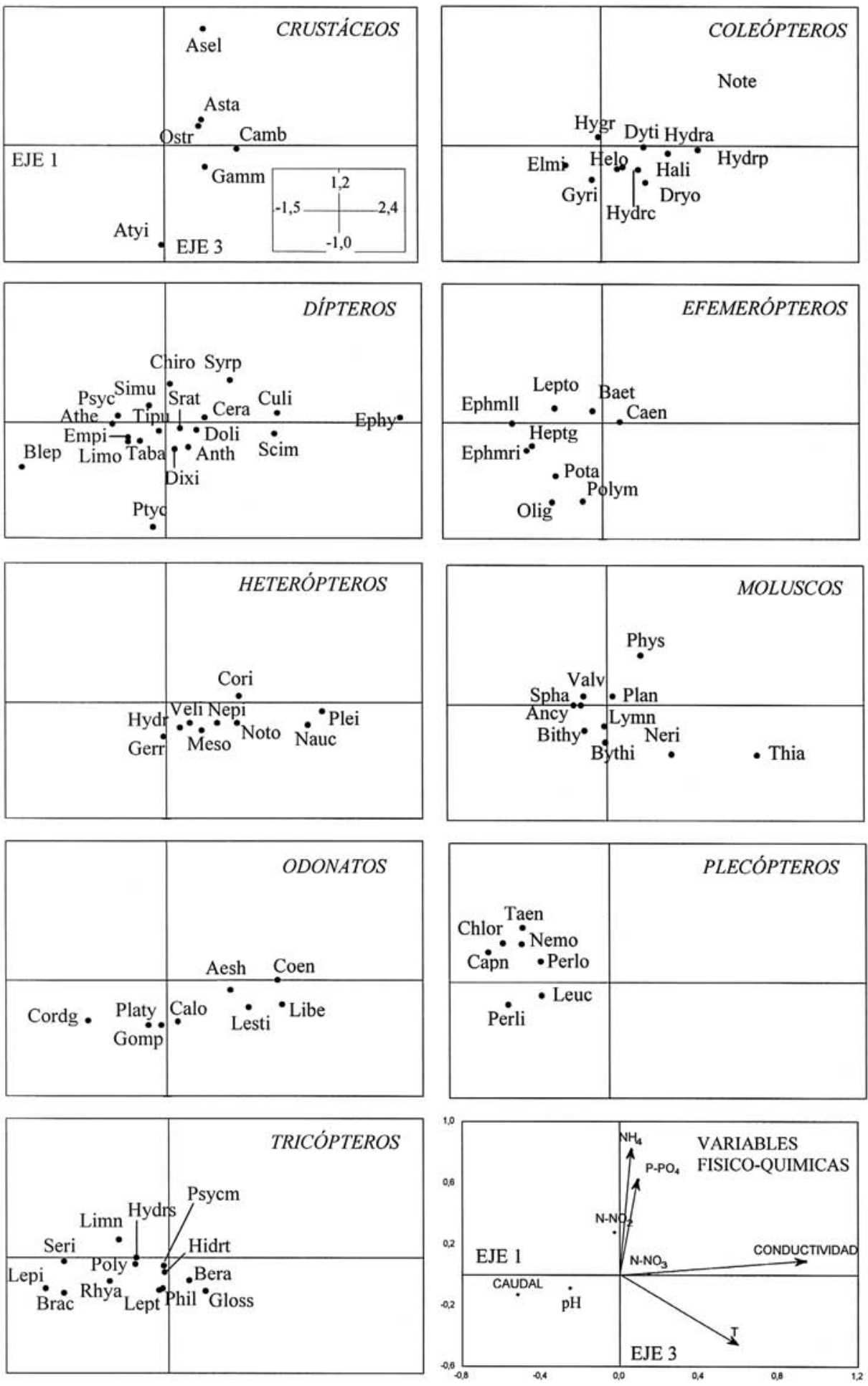

Figura 4. Representación de las mismas variables que para la figura 2 aunque en el espacio determinado por las dimensiones canónicas 1 y 3. Plot showing the same variables as for figure 2 but in the plane defined by the canonical dimensions 1 and 3. 
Las familias más halo-termófilas, según su posición relativamente extrema en el semieje positivo de este gradiente ambiental, son Thiaridae y Ephydridae, moluscos y dípteros respectivamente, que suelen citarse asociadas a estas condiciones en otras regiones mediterráneas (Chergui \& Patte, 1992; Heller \& Abotbol, 1997; Tachet et al., 2000). Aunque en menor medida que las anteriores familias, en general, crustáceos y los taxones del grupo $\mathrm{OCH}$, odonatos, coleópteros y heterópteros, pueden ser calificados de halo-termófilos sensu lato; la riqueza de familias de estos se correlacionó positivamente con esta dimensión ambiental. Abundantes familias, $\mathrm{u}$ otros taxones subordinados, de estos grupos, se han citado previamente como halotolerantes (eurihalinas), algunas halofilas, y generalmente termófilas, en ríos y ramblas mediterráneas con aguas fuertemente mineralizadas e incluso hipersalinas (Ortega et al., 1991; Gallardo et al., 1995; Guerrero et al., 1996; Moreno et al., 1997).

Las tendencias contrapuestas antes comentadas de los distintos grupos de macroinvertebrados, en cuanto a su distribución a lo largo de la dimensión salinidad-termicidad, tienen como resultado neto que este gradiente no condicione la riqueza total de familias. Las familias pertenecientes a los grupos EPT, que presentan alta riqueza en el extremo negativo de esta dimensión, son reemplazadas hacia el extremo positivo por familias pertenecientes a los grupos OCH y crustáceos. Williams et al. (1990) comentan que existe una tendencia universal de correlación negativa entre el número de taxones de cualquier ecosistema acuático y la salinidad de éste, cuando se consideran amplios rangos de esta variable (300-300000 mg/l), aunque la consideración de rangos de salinidad más estrechos suele redundar en una dilución de esta relación. En la región estudiada las ramblas hipersalinas pueden llegar a concentraciones salinas superiores a $50 \mathrm{~g} / 1$, además de temperaturas superiores a $30^{\circ} \mathrm{C}$ durante el estío, condiciones naturales que en buena medida deben contribuir a la baja riqueza de familias que suelen presentar (alrededor de 11). Quizás el esca- so peso que representan estos hábitats hipersalinos en el total de la masa de datos analizada impide obtener la relación antes comentada entre riqueza y salinidad. En cualquier caso, nuestros resultados muestran que dentro del rango de salinidad en el que se sitúan la mayoría de localidades estudiadas, valores de esta condición inferiores o iguales a $5 \mathrm{mS} / \mathrm{cm}$ no parece afectar negativamente a la riqueza de familias de macroinvertebrados

\section{Gradientes ambientales con influencia antrópica}

El caudal y la concentración de nutrientes indicadores de contaminación orgánica puntual, son variables que también condicionan los patrones de distribución de los macroinvertebrados en el conjunto de las cuencas estudiadas. El caudal genera un gradiente ambiental que se puede relacionar también con la temporalidad del medio acuático ya que las localidades con valores más bajos de caudal, en particular las situadas en los tramos medio-bajos de las cuencas más xéricas del sureste, suelen presentar un marcado estiaje, secándose por completo o permaneciendo como un rosario de pozas durante los meses secos. Los bajos caudales, la temporalidad o la intermitencia son rasgos naturales sobresalientes de los sistemas fluviales mediterráneos y, en general, de todos aquellos enmarcados por condiciones climáticas de cierta aridez (Davies et al., 1994). No obstante, en las regiones con clima mediterráneo, y sobre todo en la cuenca del Mediterráneo, a las condiciones geo-climáticas propiciatorias de tal régimen de caudal hay que sumar una larga historia y un activo presente de alteraciones antrópicas de éste, principalmente para uso agrícola y para abastecimiento de poblaciones (Gasith \& Resh, 1999). En la región estudiada son frecuentes, y a veces intensas, las alteraciones del régimen natural de caudal como consecuencia de la regulación por embalses y procesos de detracción, extracción y derivación de caudales para usos principalmente agrícolas (Robles et al., este volumen). Varios estudios muestran importantes 
alteraciones de las comunidades de macroinvertebrados como consecuencia de la regulación por embalses y reducciones de caudal en ríos mediterráneos (e.g., Tuch \& Gasith, 1989; Prat \& Ward, 1994; Muñoz \& Prat, 1996; Casas et al., 2001). Nuestros resultados muestran que, principalmente, algunas familias de efemerópteros, moluscos y los crustáceos Athyidae se asocian claramente con altos caudales (extremo positivo de la dimensión). Otras familias de efemerópteros (Leptophlebidae), dípteros (Blepharoceridae), tricópteros (Limnephilidae) y la mayoría de plecópteros se asocian a cabeceras montanas poco mineralizadas con caudal bajo. Por otra parte, algunas familias de dípteros, coleópteros y odonatos, y la mayoría de heterópteros, tienden a decantarse claramente hacia localidades con caudal muy bajo y/o carácter temporal en las cuencas más xéricas y mineralizadas. De hecho, la riqueza $\mathrm{OCH}$ se correlacionó negativamente con este segundo gradiente. La generalidad de los componentes de estos tres órdenes presenta adultos con importantes capacidades de dispersión a través del medio terrestre lo que, además del conjunto de adaptaciones que les capacitan preferentemente para la vida en medios con poca corriente o leníticos (Williams \& Felmate, 1992; Merritt \& Cummins, 1996; Tachet et al., 2000), les permite medrar más satisfactoriamente en ríos intermitentes o temporales en comparación con otros grupos de macroinvertebrados.

Aparte de los anteriores efectos cualitativos que el caudal parece tener sobre la composición de familias, la riqueza de estas no parece estar condicionada sustancialmente por esta dimensión, aunque se observa cierta correlación negativa, esta podría ser considerada como accidental. En efecto, un buen número de localidades donde se registraron algunos de los mayores valores de caudal también presentaron claros síntomas de contaminación (tramos medios y bajos de los ríos Llobregat y Júcar); de hecho la fuerza de la mencionada correlación disminuyó cuando se suprimieron del análisis las localidades más contaminadas. Además, debió contribuir también la dificultad que entraña la toma de muestras representativas de la comunidad de macroinvertebrados en aquellas localidades con gran caudal en las que resulta difícil o imposible vadear el cauce.

Junto con la regulación y derivación de caudales, la contaminación orgánica, producida principalmente por vertidos puntuales de aguas residuales de poblaciones o actividades ganaderas, suele ser la perturbación antropogénica más frecuente en ríos mediterráneos, cuyos efectos sobre la biota fluvial se multiplican cuando la dilución del efluente contaminante es pequeña al producirse en cauces con bajo caudal o temporales (e.g., Zamora-Muñoz \& Alba-Tercedor, 1996; Prat et al., 1996; Gasith \& Resh, 1999; Aguiar et al., 2002). Esta importancia se pone de manifiesto en el presente estudio, ya que la tercera dimensión resultante en el análisis canónico de correspondencias se asocia claramente a la contaminación. Asellidae, Syrphidae, Chironomidae y Physidae son las familias que parecen presentar mayor tolerancia a la contaminación en la región estudiada. Otros estudios ya han subrayado a éstas como ampliamente tolerantes al enriquecimiento orgánico del medio fluvial (e.g., Johnson et al., 1993; Zamora-Muñoz \& AlbaTercedor, 1996; Thorpe \& Lloyd, 1999) o con claras preferencias de distribución por medios acuáticos eutróficos (e.g., Tachet et al., 2000). Tradicionalmente, los plecópteros se han considerado entre los taxones más oligosaprobiontes, lo que ha llevado a clasificarlos entre los mejores indicadores de buena calidad del agua (e.g., Hynes, 1960; Hellawell, 1986; Johnson et al., 1993; Prat \& Ward, 1994; Shieh et al, 1999). No obstante, las familias de plecópteros Taenopterigidae, Nemouridae y Chloroperlidae, parecen mostrar aquí cierta tolerancia a la contaminación. Este resultado tiene ya algunos precedentes en estudios de otros ríos ibéricos, como la tolerancia de algunos Nemouridae y Leuctridae a contaminación leve en la cuenca del Río Genil (ZamoraMuñoz \& Alba-Tercedor, 1992; 1996), o el amplio rango de concentración de amonio y materia orgánica tolerado por algunas especies de plecópteros en dos ríos de Cádiz (Prenda \& Gallardo, 1999), siempre que el medio acuático 
satisfaga unos requisitos mínimos de temperatura relativamente baja, buena oxigenación y turbulencia. Algunas familias, Atyidae, Oligoneuridae, Polymitarcidae y Ptychopteridae, presentaron posiciones extremas en el semieje negativo de esta dimensión, lo que sugiere un alto valor como indicadores de muy buena calidad. Sin embargo, el contraste de este resultado con las tolerancias de tales familias a la contaminación orgánica o eutrofia expuestas por otros autores (Johnson et al., 1993; Zamora-Muñoz \& Alba-Tercedor, 1996; Tachet et al., 2000) muestra importantes divergencias. Por ejemplo, mientras que Johnson et al. (1993) citan a la única especie de la familia Atyidae como característica de aguas betamesosapróbicas, Tachet et al. (2000) la asocian claramente a medios eutróficos. Tal vez resulte conveniente subrayar que esta dimensión está principalmente relacionada con la concentración de amonio y fosfato, dos buenos indicadores en sistemas fluviales de contaminación orgánica puntual y próxima espacio-temporalmente, pero que no son necesariamente indicadores de eutrofia (Dodds, 2003). En cualquier caso, las anteriores familias asociadas aquí con aguas limpias son en general muy poco frecuentes (salvo Atyidae, que se censó en 28 muestras) por lo que el presente resultado debe ser tomado con cautela.

La gran mayoría de familias se distribuyen en posiciones intermedias de la dimensión contaminación, a pesar de que algunas de éstas (especialmente, algunos dípteros y tricópteros) se citan con requerimientos por aguas exentas de contaminación orgánica u oligotróficas (ver Johnson et al., 1993; Tachet et al. 2000) o se les asigna un alto valor indicador de buena calidad en algunos índices bióticos (e.g., JáimezCuéllar et al., este volumen). Quizás las peculiares características de nuestro universo muestral, y probablemente de muchos otros sistemas fluviales de la cuenca del Mediterráneo, donde suelen dominar fuertes pendientes a lo largo de la mayor parte del perfil longitudinal, permiten mantener condiciones de turbulencia y oxigenación del agua suficientemente aceptables para ciertos taxones frente a eventos no extremos de contaminación orgánica, al menos cuando el caudal lo permita. Debemos tener en cuenta que las concentraciones de amonio y fosfato en el agua son indicadores de contaminación, pero las consecuencias reales para los organismos de un vertido de aguas residuales dependerán no sólo de la naturaleza del vertido sino también del resultado de su interacción con numerosos factores y procesos del ecosistema fluvial.

La riqueza de familias total y la de todos los grupos taxonómicos en particular (crustáceos, moluscos, dípteros, EPT y $\mathrm{OCH}$ ) se muestra aquí condicionada negativamente de forma neta por la contaminación. El empobrecimiento de la comunidad de macroinvertebrados por contaminación orgánica es un patrón extensamente documentado, que ofrece poca discusión cualquiera que sea la resolución taxonómica utilizada (ver Rosenberg \& Resh, 1993). También es cierto que se ha descrito cierto incremento de la riqueza de invertebrados a consecuencia de una ligera fertilización (eutrofización), especialmente cuando ocurre en tramos fluviales muy oligotróficos, no obstante, en sistemas fluviales claramente eutrofizados la comunidad bentónica sufre un neto empobrecimiento taxonómico (Giller \& Malmqvist, 1999).

\section{Implicaciones para la evaluación de la calidad biológica}

La evaluación biológica de la calidad de los ecosistemas fluviales y el ajuste de sus fundamentos teóricos y metodológicos a las particulares de cada región o país constituye un importante tema de debate desde hace más de dos décadas (ver Rosenberg \& Resh, 1993; Karr \& Chu, 1999; Griffith et al., 2001). Actualmente, en la Península Ibérica los índices biológicos fundamentados en macroinvertebrados y más utilizados para la evaluación de la calidad de sistemas fluviales se basan en la resolución taxonómica a familia y en la asignación de una puntuación de acuerdo con el valor indicador a cada taxón: son IBMWP, suma de las puntuaciones de las familias censadas, e IASPT, puntuación media de las familias censadas (Alba-Tercedor \& Pujante, 2000). Nuestros 
resultados muestran considerable correlación negativa de ambos índices con la dimensión contaminación orgánica, lo que pone de manifiesto cierta bondad en su capacidad de evaluación de la calidad del agua, especialmente del índice IBMWP. El índice IASPT, además de presentar un menor valor absoluto de correlación con esta dimensión, mostró una muy fuerte correlación negativa con la dimensión salinidadtermicidad, algo más del doble de la mostrada por el índice IBMWP. Considerando que los caracteres salino y/o térmico de los ríos en nuestra región parecen tener una fuerte causa natural, como se discutió anteriormente, la mencionada correlación no resulta deseable y advierte de la cautela con la que debemos interpretar los resultados proporcionados por este índice (IASPT), al menos en ríos mediterráneos. En esta notable flaqueza del índice IASPT probablemente subyace un importante desajuste en la puntuación del valor indicador adjudicado a varias familias, que, en general, se puede resumir con la tendencia a sobrevalorar al grupo taxonómico EPT frente al OCH. Como sugieren los resultados aquí presentados, bastantes familias EPT no parecen merecer el alto valor indicador de buena calidad que se les atribuye, mientras que algunas familias componentes del grupo $\mathrm{OCH}$ podrían estar infravaloradas. En la medida que el índice IBMWP está condicionado por las puntuaciones asignadas a cada familia, sus evaluaciones sufrirán también las mencionadas flaquezas. No obstante, este índice también cuenta con la riqueza de familias y esto claramente compensa y mejora sus resultados, por ser ésta una variable no correlacionada con el gradiente salinidad-termicidad. Una consecuencia inmediata derivada de tal sesgo es una marcada tendencia a la penalización de los tramos fluviales más térmicos y mineralizados, que para alcanzar puntuaciones equivalentes a tramos no contaminados de agua "dulce-fría" deben mostrar una sobresaliente riqueza de familias. Por tanto, a pesar del esfuerzo realizado para la adaptación de estos índices a las particularidades faunísticas ibéricas (ver AlbaTercedor Pujante, 2000), si se pretende que los índices se comporten como herramientas precisas de evaluación y de alerta rápida ante los primeros síntomas de estrés ambiental antropogénico (Karr \& Chu, 1999; Wright, 2000), parece recomendable más investigación que incluya tanto la prospección de nuevas áreas geográficas así como la realización de algunos estudios experimentales de laboratorio y campo, para obtener evidencias fuertes sobre el valor indicador de los taxones.

\section{AGRADECIMIENTOS}

Este trabajo se ha realizado mediante la financiación de los proyectos HID98-0323-C05 y REN2001-3438-C07 del Ministerio de Ciencia y Tecnología y PLP/10/FS/97 de la Fundación Séneca de la CARM. Nuestro especial agradecimiento al Area de Coordinación y Aplicaciones Tecnológicas de la D.G.O.H. del Ministerio de Medio Ambiente, al Àrea de Medi Ambient de la Diputació de Barcelona, a la Delegación de Granada de la Consejería de Medio Ambiente de la Junta de Andalucía y a la Agencia Catalana del Aigua por su apoyo.

\section{BIBLIOGRAFÍA}

AGUiAR, F. C., M. T. FERREIRA \& P. PINTO. 2002. Relative influence of environmental variables on macroinvertebrate assemblages from an Iberian basin. J. N. Am. Benthol. Soc., 21: 43-53.

ALBA-TERCEDOR, J., G. GONZÁlEZ \& M. A. PUIG. 1992. Present level of knowledge regarding fluvial macroinvertebrate communities in Spain. Limnetica, 8: 231-241.

ALBA-TERCEDOR, J. \& A. PUJANTE. 2000. Running-water biomonitoring in Spain. Opportunities for a predictive approach. In: Assessing the Biological Quality of Freshwater: RIVPACS and similar techniques. J.F. Wright, D.W. Sutcliffe \& M. Furse (eds.): 207-216. Freshwater Biological Association.

ALBA-TERCEDOR, J. \& A. SÁNCHEZ-ORTEGA. 1988. Un método rápido y simple para evaluar la calidad biológica de las aguas corrientes basado en el de Hellawell (1978). Limnetica, 4: 51-56. 
ALBA-TERCEDOR, J. \& N. PRAT. 1992. Spanish experience in the use of macroinvertebrates as biological pollution indicators. In: River water quality: ecological assessment and control. P.J. Newman, M.A. Piavaux \& R.A. Sweeting (eds.): 733-738. Publication EUR 14606 EN-FR, Commision of the European Communities, Luxembourg.

ALBA-TERCEDOR, J. 1996. Macroinvertebrados acuáticos y calidad de las aguas de los ríos. $I V$ Simposio sobre el Agua en Andalucía, II: 203-213.

CAPEL MOLINA, J. J. 2000. El clima de la Península Ibérica. España: Ariel Geografía. S.A.

CASAS, J. J., C. ZAMORA-MUÑOZ, F. ARCHILA \& J. ALBA-TERCEDOR. 2001. The effect of a headwater dam on the use of leaf bags by invertebrate communities. Regul. Riv., 16: 577-591.

CHERGUI, H. \& E. PATTE. 1992. Processing of fresh and dry Salix leaves in a Maroccan river system. Acta Oecologica, 13: 291-298.

COLLIER, K. J. 1995. Environmental factors affecting the taxonomic composition of aquatic macroinvertebrate communities in lowland waterways of Northland, New Zealand. New Zeal. J. Mar. Freshwat. Res., 29: 453-465.

CORTES, R. V., M. T. FERREIRA \& F. N. GODINHO. 1998. Benthic community organization in an intermittent lotic system. Verh. int. Ver. Limnol., 26: 1002-1007.

DAVIES, B. R., M. C. THOMS, K. F. WALKER, J. H. O'KEEFFE \& J. A. GORE. 1994. Dryland rivers: their ecology, conservation and management. In: The Rivers Handbook vol. 2. P. Calow \& G.E. Petts (eds.): 484-512. Blackwell Scientific, Oxford.

D.O.C.E. 2000. Directiva 2000/60/CE del Parlamento Europeo y del Consejo de 23 de octubre de 2000 por la que se establece un marco comunitario de actuación en el ámbito de la politica de aguas. D.O.C.E. L 327 de 22.12.00. 69 pp.

DOODS, W., 2003. Misuse of inorganic N and soluble reactive $\mathrm{P}$ concentrations to indicate nutrient status of surface waters. J. N. Am. Benthol. Soc., 22: 171-181.

GALLARDO, A., J. FRESNEDA \& J. TOJA. 1995. Distribución de los coleópteros acuáticos (Insecta, Coleoptera) en dos cuencas del sur de la P. Ibérica. Relaciones con algunos factores del medio. Limnetica, 11: 19-28.

GASITH, A. \& V. H. RESH. 1999. Streams in Mediterranean climate regions: Abiotic influences and biotic responses to predictable seasonal events. Ann. Rev. Ecol. Syst., 30: 51-81.

GILLER, P. S. \& B. MALMQVIST. 1998. The Biology of streams and rivers. Oxford: Oxford University Press.

GRIFFITH, M. B., P. R. KAUFFMANN, A. T. HERLIHY \& B. H. HILL. 2001. Analysis of macroinvertebrate assemblages in relation to environmental gradients in Rocky Mountain streams. Ecological Applications, 11: 489-505.

GUERRERO, C., A. MILLAN, J. VELASCO, J.L. MORENO, M. L. SUÁREZ \& M. R. VIDALABARCA. 1996. Aproximación al conocimiento de la dinámica espacio-temporal de la comunidad de invertebrados acuáticos en un tramo de río de características semiáridas (río Chicamo: cuenca del río Segura). 125 Aniversario de la RSEHN, tomo extraordinario: 99-102.

HARPER, P. P. 1979. Plecoptera. In: Canada and its Insect Fauna. H. V. Danks (ed.): 311-313. Mem. Ent. Soc. Can., 108: 1-573

HELLAWELL, J. M. 1986. Biological indicators of freshwater pollution and environmental management. London \& New York: Pollution Monitoring Series. Elsevier Applied Science.

HELLER, J. \& A. ABOTBOL. 1997. Litter shredding in a desert oasis by the snail Melanopsis praemorsa. Hydrobiologia, 344: 65-73.

HILDREW, A. G. 1992. Food webs and species interactions. In: The River Handbook. P. Caloww \& G.E. Petts (eds.): 309-330. Blackwell Scientific Publications, Oxford.

HYNES, H. B. N. 1960. The Biology of Polluted Waters. Liverpool: Liverpool University Press.

JÁIMEZ-CUÉLLAR, P., S. VIVAS, N. BONADA, S. ROBLES, A. MELLADO, M. ÁlVAREZ, J. AVILÉS, J. CASAS, M. ORTEGA, I. PARDO, N. PRAT, M. RIERADEVALL, C.E. SÁINZCANTERO, A. SÁNCHEZ-ORTEGA, M. L. SUÁREZ, M. TORO, M. R. VIDAL-ABARCA, C. ZAMORA-MUÑOZ \& J. ALBA-TERCEDOR. 2002. Protocolo GUADALMED (PRECE). Limnetica, 21: 187-204.

JOHNSON, R. K., T. WIEDERHOLM \& D. M. ROSENBERG. 1993. Freshwater biomonitoring using individual organisms, populations, and species assemblages of benthic macroinvertebrates. In: Freshwater biomonitoring and benthic macroinvertebrates. D.M. Rosenberg \& V.H. Resh (eds.): 40-158. Chapman \& Hall, New York, USA. 
KARR, J.R. \& E.W. CHU. 1999. Restoring life in running waters. Better biological monitoring. Washington DC: Island Press.

KAY, W.R., S. A. HALSE, M. D. SCANLON \& M. J. SMITH. 2001. Distribution and environmental tolerances of aquatic macroinvertebrate families in the agricultural zone of southwestern Australia. J.N. Am. Benthol. Soc. , 20: 182-199.

LAMPERT, W. \& U. SOMMER. 1997. Limnoecology. The ecology of lakes and streams. New York: Oxford University Press.

LELAND, H. V. \& S. V. FEND. 1998. Benthic invertebrate distributions in the San Joaquin river, California, in relation to physical and chemical factors. Can. J. Fish. Aquat. Sci., 55: 1051-1067.

MARCHANT, R., A. HIRST, R. NORRIS \& L. METZELING. 1999. Classification of macroinvertebrate communities across drainage basins in Victoria, Australia: consequences of sampling on a broad spatial scale for predictive modelling. Freshwat. Biol., 41:253-268.

MERRITT, R. W. \& K. W. CUMMINS. 1996. An introduction to the aquatic insects of North America, $3^{\text {rd }}$ ed. R.W. Merritt \& K.W. Cummins (eds.): 309-349. Kendal/Hunt Publishing Company, Dubuque, Iowa.

MORENO, J. L., A. MILLÁN, M. L. SUÁREZ, M. R. VIDAL-ABARCA \& J. VELASCO. 1997. Aquatic coleoptera and heteroptera assemblages in waterbodies from ephemeral coastal streams ("ramblas") of South-eastern Spain. Arch. Hydrobiol., 141: 93-107.

MUÑOZ, I. \& N. PRAT. 1996. Effects of water abstraction and pollution on macroinvertebrate community in a Mediterranean river. Limnetica, 12: 916.

NATIONAL WATER COUNCIL. 1981. River Quality. The 1980 survey and future outlook. NWC, London.

ORTEGA, M., M. L. SUÁREZ, M. R. VIDALABARCA \& L. RAMÍREZ-DÍAZ. 1991. Aspectos dinámicos de la composición y estructura de la comunidad de invertebrados acuáticos de la Rambla del Moro después de una riada (Cuenca del Río Segura: SE de España). Limnetica, 7: 11-24.

PLAFKIN, J. L., M. T. BARBOUR, K. D. PORTER, S. K. GROSS \& R. M. HUGHES. 1989. Rapid Bioassessment protocols for use in streams and rivers: benthic macroinvertebrates and fish. EPA/444/4-89-001. United Estates Environmental Protection Agency, Washington D. C., USA.
PRAT, N. \& J. WARD. 1994. The tamed river. In: Limnology Now: A paradigm of planetary problems. R. Margalef (ed.): 219-236. Elsevier, Amsterdam.

PRAT, N., M. RIERADEVALL \& A. MUNNÉ. 1996. La qualitat ecologica del Besòs i el Llobregat, Estudis de la qualitat ecologica dels rius: I (Informe 1994-1995). Area del Medi Ambient de la Diputació de Barcelona.

PRENDA, J. \& A. GALLARDO-MAYENCO. 1999. Distribution patterns, species assemblages and habitat selection of the stoneflies (Plecoptera) from two Mediterranean river basins in Southern Spain. Internat. Rev. ges. Hydrobiol., 84: 595-608.

ROBLES, S., M. TORO, C. NUÑO, J. AVILÉS, J. ALBA-TERCEDOR, M. ÁLVAREZ, N. BONADA, J. CASAS, P. JÁIMEZ-CUÉLLAR, A. MELLADO, A. MUNNÉ, I. PARDO, N. PRAT, M. L. SUÁREZ, M. R. VIDAL-ABARCA, S. VIVAS, G. MOYÁ \& G. RAMON. 2002. Descripción de las cuencas mediterráneas seleccionadas en el proyecto GUADALMED. Limnetica, 21: 35-61.

ROSENBERG, D. M. \& V. H. RESH. 1993. Freshwater biomonitoring and benthic macroinvertebrates. New York: Chapman \& Hall.

SHIEH, S. H., B. C. KONDRATIEFF, J. V. WARD \& D. A. RICE. 1999. The relationship of macroinvertebrate assemblages to water chemistry in a polluted Colorado plains stream. Arch. Hydrobiol., 145: 405-432.

STEWART, K. W. \& B. P. STARK. 1988. Nymphs of North American stonefly genera (Plecoptera). Thomas Say Found. Ser. Ent. Soc. Am., 12: 1460.

SUÁREZ, M. L., M. R. VIDAL-ABARCA, M. M. SÁNCHEZ-MONTOYA, J. ALBA-TERCEDOR, M. ÁlVAREZ, J. AVILÉS, N. BONADA, J. CASAS, P. JÁIMEZ-CUÉLLAR, A. MUNNÉ, I. PARDO, N. PRAT, M. RIERADEVALL, M.J. SALINAS, M. TORO \& S. VIVAS. 2002. Las riberas de los ríos mediterráneos y su calidad: El uso del índice QBR. Limnetica, 21: 135-148.

TACHET, H., P. RICHOUX, M. BOURNAUD \& P. USSEGLIO-POLATERA. 2000. Invertébrés d'eau douce. Systématique, biologie, écologie. Paris: CNRS Editions.

TER BRAAK, C. J. F. 1986. Canonical correspondence analysis: a new eigenvector technique for multivariate direct gradient analysis. Ecology, 67: 1167-1179. 
TER BRAAK, C.J.F. \& P. SMILAUER. 1998. CANOCO Reference Manual and User's Guide to Canoco for Windows: Software for Canonical Community Ordination (version 4). Microcomputer Power. Ithaca, NY.

THORPE, T. \& B. LLOYD. 1999. The macroinvertebrate fauna of St. Lucia elucidated by canonical correspondence análisis. Hidrobiologia, 400: 195-203.

TORO, M., S. ROBLES, J. AVILÉS, C. NUÑO, S. VIVAS, N. BONADA, N. PRAT, J. ALBATERCEDOR, J. CASAS, C. GUERRERO, P. JÁIMEZ-CUÉLLAR， J. L. MORENO, G. MOYÁ, G. RAMON, M. L. SUÁREZ, M. R. VIDAL-ABARCA, M. ÁLVAREZ \& I. PARDO. 2002. Calidad de las aguas de los ríos mediterráneos del proyecto GUADALMED. Limnetica, 21: 63-75.

TUCH, A \& A. GASITH. 1989. Effects of an upland impoundment on structural and functional properties of a small stream in a basaltic plateau (Golan Heights, Israel). Reg. Riv., 3: 153-167.

WIBERG-LARSEN, P., K. P. BRODERSEN, S. BIRKHOLM, P. N. GRON \& J. SKRIVER. 2000. Species Richness and assemblage structure of Thricoptera in Danish streams. Freswat. Biol., 43:633-647.

WIGGINS, G. B. 1996. Trichoptera Families. In $A n$ introduction to the aquatic insects of North America, $3^{\text {rd }}$ ed. R.W. Merritt \& K.W. Cummins (eds.): 309-349. Kendal/Hunt Publishing Company, Dubuque, Iowa.

WILliAMS, D. D. \& B. W. FELTMATE. 1992. Aquatic Insects. Oxon: CAB International.

WILLIAMS, W.D. 1987. Salinization of rivers and streams: an important environmental hazard. Ambio, 16: 180-185.

WILLIAMS, W. D., A. J. BOULTON \& R. G. TAAFFE. 1990. Salinity as a determinant of salt lake fauna: a question of scale. Hydrobiologia, 197: 257-266.

WRIGHT, J. F. 2000. An Introduction to RIVPACS. In Assessing the biological quality of fresh waters. RIVPACS and other techniques. J.F. Wright, D.W. Sutcliffe \& M.T. Furse (eds.): 1-24. Freshwater Biological Association, Ambleside, UK.

WRIGHT, J. F., D. W. SUTCLIFFE \& M. T. FURSE. 2000. Assessing the biological quality of fresh waters. RIVPACS and other techniques. Ambleside: Freshwater Biological Association.

ZAMORA MUÑOZ, C. \& ALBA-TERCEDOR, J. 1992. Caracterización y calidad de las aguas del río Monachil (Sierra Nevada). Factores fisicoquímicos y comunidades de macroinvertebrados acuáticos. Junta de Andalucía, Agencia de Medio Ambiente. Granada.

ZAMORA-MUÑOZ, C. \& ALBA-TERCEDOR, J. 1996. Bioassessment of organically polluted rivers using biotic indices and multivariate methods. $J$. N. Am. Benthol. Soc., 15: 332-352. 
Anexo 1. Listado de taxones (principalmente familias) identificados en las 12 cuencas fluviales estudiadas. Se indica para cada cuenca estudiada la presencia (1) o ausencia (0) de las familias. En la columna de códigos se indica la abreviatura utilizada para cada taxon en las figuras 2 y 4. List of taxa (mainly families) recorded in the 12 fluvial basins studied. Presence (1) or absence (0) of the families is indicated for each basin. The column of codes indicates the abbreviations used for each taxa in figures 2 and 4.

\section{TAXON}

Código Adra Aguas Almanzora Besos Guadalfeo Júcar Llobregat Mijares Pollença Segura Sóller Turia

\begin{tabular}{|c|c|c|c|c|c|c|c|c|c|c|c|c|c|}
\hline Aeshnidae & Aesh & 1 & 1 & 1 & 1 & 0 & 1 & 1 & 1 & 1 & 1 & 1 & 1 \\
\hline Ancylidae & Ancy & 1 & 1 & 1 & 1 & 1 & 1 & 1 & 1 & 1 & 1 & 1 & 1 \\
\hline Anthomyidae & Anth & 1 & 1 & 1 & 1 & 1 & 1 & 1 & 1 & 1 & 1 & 1 & 0 \\
\hline Asellidae & Asel & 0 & 0 & 0 & 1 & 1 & 1 & 1 & 0 & 1 & 0 & 1 & 0 \\
\hline Astacidae & Asta & 0 & 0 & 0 & 1 & 0 & 0 & 1 & 1 & 0 & 1 & 1 & 0 \\
\hline Athericidae & Athe & 1 & 0 & 0 & 1 & 1 & 1 & 1 & 1 & 0 & 1 & 0 & 1 \\
\hline Atyidae & Atyi & 0 & 0 & 0 & 0 & 0 & 1 & 0 & 0 & 0 & 1 & 0 & 1 \\
\hline Baetidae & Baet & 1 & 1 & 1 & 1 & 1 & 1 & 1 & 1 & 1 & 1 & 1 & 1 \\
\hline Beraeidae & Bera & 0 & 0 & 0 & 1 & 0 & 0 & 1 & 0 & 0 & 1 & 0 & 0 \\
\hline Bithyniidae & Bith & 0 & 0 & 0 & 1 & 0 & 1 & 1 & 0 & 0 & 0 & 0 & 1 \\
\hline Blephariceridae & Blep & 1 & 0 & 0 & 0 & 1 & 0 & 0 & 0 & 0 & 0 & 0 & 0 \\
\hline Brachycentridae & Brac & 1 & 0 & 1 & 0 & 1 & 1 & 1 & 0 & 0 & 1 & 0 & 0 \\
\hline Bythinellidae & Byth & 1 & 0 & 0 & 1 & 0 & 1 & 1 & 1 & 0 & 0 & 0 & 1 \\
\hline Caenidae & Caen & 1 & 1 & 1 & 1 & 1 & 1 & 1 & 1 & 1 & 1 & 1 & 1 \\
\hline Calamoceratidae & Cala & 0 & 0 & 0 & 0 & 0 & 0 & 0 & 0 & 0 & 1 & 0 & 0 \\
\hline Calopterygidae & Calo & 1 & 1 & 1 & 1 & 1 & 1 & 1 & 1 & 0 & 1 & 0 & 1 \\
\hline Cambaridae & Camb & 0 & 1 & 0 & 1 & 0 & 1 & 1 & 1 & 0 & 1 & 0 & 1 \\
\hline Capniidae & Capn & 1 & 0 & 1 & 0 & 1 & 0 & 0 & 0 & 0 & 1 & 0 & 0 \\
\hline Ceratopogonidae & Cera & 1 & 1 & 1 & 1 & 1 & 1 & 1 & 1 & 1 & 1 & 1 & 1 \\
\hline Chironomidae & Chir & 1 & 1 & 1 & 1 & 1 & 1 & 1 & 1 & 1 & 1 & 1 & 1 \\
\hline Chloroperlidae & Chlo & 1 & 0 & 1 & 1 & 1 & 0 & 1 & 0 & 0 & 0 & 0 & 0 \\
\hline Chrysomelidae & Chry & 0 & 0 & 0 & 0 & 0 & 0 & 0 & 0 & 1 & 0 & 1 & 0 \\
\hline Cirolanidae & Ciro & 0 & 0 & 0 & 0 & 0 & 0 & 0 & 0 & 0 & 1 & 0 & 0 \\
\hline Cladocera & Clad & 0 & 0 & 0 & 1 & 0 & 0 & 1 & 0 & 0 & 1 & 0 & 0 \\
\hline Coenagrionidae & Coen & 0 & 1 & 1 & 1 & 0 & 1 & 1 & 1 & 1 & 1 & 0 & 1 \\
\hline Cordulegasteridae & Cord & 1 & 0 & 1 & 1 & 1 & 1 & 1 & 1 & 1 & 0 & 0 & 1 \\
\hline Corduliidae & Cord & 0 & 0 & 0 & 1 & 0 & 0 & 0 & 0 & 0 & 1 & 0 & 0 \\
\hline Corixidae & Cori & 1 & 1 & 1 & 1 & 0 & 1 & 1 & 1 & 1 & 1 & 1 & 1 \\
\hline Culicidae & Culi & 1 & 1 & 1 & 1 & 1 & 0 & 1 & 0 & 1 & 1 & 1 & 1 \\
\hline Curculionidae & Curc & 0 & 1 & 1 & 0 & 0 & 0 & 0 & 0 & 0 & 1 & 0 & 0 \\
\hline Dixidae & Dixi & 1 & 1 & 1 & 1 & 1 & 1 & 1 & 0 & 1 & 1 & 1 & 1 \\
\hline Dolichopodidae & Doli & 0 & 1 & 1 & 1 & 1 & 0 & 1 & 1 & 1 & 1 & 1 & 0 \\
\hline Dryopidae & Dryo & 1 & 1 & 1 & 1 & 1 & 1 & 1 & 1 & 1 & 1 & 1 & 1 \\
\hline Dugesiidae & Duge & 1 & 0 & 0 & 1 & 1 & 1 & 1 & 1 & 1 & 1 & 1 & 1 \\
\hline Dytiscidae & Dyti & 1 & 1 & 1 & 1 & 1 & 1 & 1 & 1 & 1 & 1 & 1 & 1 \\
\hline Ecnomidae & Ecno & 0 & 0 & 0 & 0 & 0 & 0 & 1 & 0 & 0 & 0 & 0 & 1 \\
\hline Elmidae & Elmi & 1 & 1 & 1 & 1 & 1 & 1 & 1 & 1 & 1 & 1 & 0 & 1 \\
\hline Empididae & Empi & 1 & 1 & 1 & 1 & 1 & 1 & 1 & 1 & 0 & 1 & 1 & 1 \\
\hline Ephemerellidae & Ephmll & 1 & 0 & 1 & 1 & 1 & 1 & 1 & 1 & 0 & 1 & 0 & 1 \\
\hline Ephemeridae & Ephmri & 1 & 0 & 1 & 1 & 1 & 1 & 1 & 1 & 0 & 1 & 0 & 1 \\
\hline Ephydridae & Ephy & 0 & 0 & 0 & 1 & 0 & 0 & 1 & 0 & 1 & 1 & 1 & 0 \\
\hline Erpobdellidae & Erpo & 1 & 1 & 1 & 1 & 1 & 1 & 1 & 1 & 1 & 1 & 1 & 1 \\
\hline Ferrissiidae & Ferr & 0 & 0 & 0 & 0 & 0 & 0 & 1 & 0 & 0 & 0 & 0 & 0 \\
\hline Gammaridae & Gamm & 1 & 1 & 1 & 1 & 1 & 1 & 1 & 1 & 1 & 1 & 1 & 1 \\
\hline Georissidae & Geor & 0 & 0 & 0 & 0 & 0 & 0 & 0 & 0 & 0 & 1 & 0 & 0 \\
\hline Gerridae & Gerr & 1 & 1 & 1 & 1 & 1 & 1 & 1 & 1 & 1 & 1 & 0 & 1 \\
\hline Glossiphoniidae & Glossi & 1 & 1 & 1 & 1 & 1 & 1 & 1 & 1 & 1 & 1 & 1 & 1 \\
\hline
\end{tabular}


Anexo 1. Continuación. Continuation.

TAXON

Código Adra Aguas Almanzora Besos Guadalfeo Júcar Llobregat Mijares Pollença Segura Sóller Turia

\begin{tabular}{|c|c|c|c|c|c|c|c|c|c|c|c|c|c|}
\hline Glossosomatidae & Gloss & 1 & 1 & 1 & 1 & 1 & 1 & 0 & 1 & 1 & 1 & 0 & 1 \\
\hline Goeridae & Goer & 0 & 0 & 0 & 0 & 0 & 0 & 1 & 0 & 0 & 0 & 0 & 0 \\
\hline Gomphidae & Gomp & 1 & 1 & 1 & 1 & 1 & 1 & 1 & 1 & 0 & 1 & 0 & 1 \\
\hline Gyrinidae & Gyri & 1 & 1 & 1 & 1 & 1 & 1 & 1 & 1 & 1 & 1 & 1 & 1 \\
\hline Haliplidae & Hali & 1 & 1 & 1 & 1 & 1 & 1 & 1 & 1 & 1 & 1 & 1 & 1 \\
\hline Hebridae & Hebr & 0 & 1 & 0 & 0 & 0 & 0 & 0 & 0 & 0 & 1 & 0 & 0 \\
\hline Helodidae & Helo & 1 & 1 & 1 & 1 & 1 & 1 & 1 & 1 & 0 & 1 & 0 & 1 \\
\hline Helophoridae & Helop & 1 & 0 & 0 & 0 & 1 & 0 & 0 & 0 & 0 & 1 & 0 & 0 \\
\hline Heptageniidae & Hept & 1 & 0 & 1 & 1 & 1 & 1 & 1 & 1 & 0 & 1 & 0 & 1 \\
\hline Heteroceridae & Hete & 0 & 0 & 0 & 0 & 0 & 0 & 0 & 0 & 0 & 1 & 0 & 0 \\
\hline Hirudidae & Hiru & 0 & 0 & 0 & 0 & 1 & 0 & 0 & 0 & 0 & 1 & 0 & 0 \\
\hline Hydracarina & Hydrac & 1 & 1 & 1 & 1 & 1 & 1 & 1 & 1 & 1 & 1 & 1 & 1 \\
\hline Hydraenidae & Hydra & 1 & 1 & 1 & 1 & 1 & 1 & 1 & 1 & 1 & 1 & 1 & 1 \\
\hline Hydridae & Hydri & 0 & 0 & 0 & 1 & 0 & 0 & 0 & 0 & 0 & 0 & 0 & 0 \\
\hline Hydrobiidae & Hydrob & 1 & 1 & 1 & 1 & 1 & 1 & 1 & 1 & 1 & 1 & 1 & 1 \\
\hline Hydrochidae & Hydrc & 0 & 0 & 0 & 0 & 0 & 0 & 0 & 0 & 1 & 1 & 0 & 0 \\
\hline Hydrometridae & Hydr & 1 & 1 & 1 & 1 & 1 & 1 & 1 & 1 & 1 & 1 & 0 & 1 \\
\hline Hydrophilidae & Hydrp & 1 & 1 & 1 & 1 & 1 & 1 & 1 & 0 & 1 & 1 & 1 & 0 \\
\hline Hydropsychidae & Hydrs & 1 & 1 & 1 & 1 & 1 & 1 & 1 & 1 & 1 & 1 & 0 & 1 \\
\hline Hydroptilidae & Hydrt & 1 & 1 & 1 & 1 & 1 & 1 & 1 & 1 & 1 & 1 & 1 & 1 \\
\hline Hygrobiidae & Hygr & 0 & 0 & 0 & 1 & 0 & 0 & 1 & 1 & 1 & 0 & 1 & 0 \\
\hline Lepidostomatidae & Lepi & 1 & 0 & 0 & 0 & 1 & 1 & 1 & 0 & 0 & 1 & 0 & 1 \\
\hline Leptoceridae & Lept & 1 & 1 & 1 & 1 & 1 & 1 & 1 & 1 & 1 & 1 & 0 & 1 \\
\hline Leptophlebiidae & Lepto & 1 & 0 & 1 & 1 & 1 & 1 & 1 & 1 & 0 & 1 & 0 & 1 \\
\hline Lestidae & Lest & 0 & 0 & 1 & 1 & 0 & 0 & 1 & 1 & 1 & 1 & 0 & 0 \\
\hline Leuctridae & Leuc & 1 & 1 & 1 & 1 & 1 & 1 & 1 & 1 & 1 & 1 & 1 & 1 \\
\hline Libellulidae & Libe & 1 & 1 & 1 & 1 & 1 & 1 & 1 & 1 & 1 & 1 & 1 & 0 \\
\hline Limnephilidae & Limn & 1 & 1 & 1 & 1 & 1 & 1 & 1 & 1 & 1 & 1 & 1 & 1 \\
\hline Limoniidae & Limo & 1 & 1 & 1 & 1 & 1 & 1 & 1 & 1 & 1 & 1 & 1 & 1 \\
\hline Lymnaeidae & Lymn & 1 & 1 & 1 & 1 & 1 & 1 & 1 & 1 & 1 & 1 & 1 & 1 \\
\hline Mesoveliidae & Meso & 0 & 1 & 1 & 1 & 0 & 1 & 1 & 0 & 0 & 1 & 0 & 1 \\
\hline Naucoridae & Nauc & 1 & 1 & 1 & 1 & 0 & 0 & 0 & 1 & 0 & 1 & 0 & 0 \\
\hline Nemouridae & Nemo & 1 & 0 & 1 & 1 & 1 & 1 & 1 & 1 & 0 & 1 & 0 & 1 \\
\hline Nepidae & Nepi & 1 & 1 & 1 & 1 & 1 & 1 & 1 & 0 & 1 & 1 & 1 & 1 \\
\hline Neritidae & Neri & 0 & 0 & 0 & 0 & 0 & 1 & 0 & 0 & 0 & 1 & 0 & 1 \\
\hline Noteridae & Note & 0 & 1 & 0 & 0 & 0 & 0 & 0 & 0 & 0 & 1 & 0 & 0 \\
\hline Notonectidae & Noto & 1 & 1 & 1 & 1 & 1 & 1 & 1 & 1 & 0 & 1 & 1 & 1 \\
\hline Ochtheridae & Octh & 0 & 0 & 0 & 0 & 0 & 0 & 0 & 0 & 0 & 1 & 0 & 0 \\
\hline Odontoceridae & Odon & 0 & 0 & 0 & 1 & 0 & 0 & 1 & 0 & 0 & 0 & 0 & 0 \\
\hline Oligochaeta & Olig & 1 & 1 & 1 & 1 & 1 & 1 & 1 & 1 & 1 & 1 & 1 & 1 \\
\hline Oligoneuriidae & Olig & 0 & 0 & 0 & 0 & 1 & 1 & 0 & 0 & 0 & 0 & 0 & 0 \\
\hline Oribatidae & Orib & 0 & 0 & 0 & 1 & 0 & 0 & 1 & 0 & 0 & 0 & 0 & 0 \\
\hline Osmylidae & Osmy & 0 & 0 & 0 & 1 & 0 & 0 & 0 & 0 & 0 & 0 & 0 & 0 \\
\hline Ostracoda & Ostr & 1 & 1 & 1 & 1 & 1 & 1 & 1 & 1 & 1 & 1 & 1 & 1 \\
\hline Palaemonidae & Pala & 0 & 0 & 0 & 0 & 0 & 0 & 0 & 0 & 0 & 0 & 0 & 1 \\
\hline Perlidae & Perli & 1 & 0 & 1 & 1 & 1 & 1 & 1 & 1 & 0 & 1 & 0 & 1 \\
\hline Perlodidae & Perlo & 1 & 0 & 1 & 1 & 1 & 1 & 1 & 0 & 0 & 1 & 0 & 1 \\
\hline Philopotamidae & Phil & 1 & 1 & 1 & 1 & 1 & 1 & 1 & 1 & 1 & 1 & 0 & 1 \\
\hline Phryganeidae & Phry & 0 & 0 & 0 & 1 & 0 & 0 & 0 & 0 & 0 & 0 & 0 & 0 \\
\hline Physidae & Phys & 1 & 1 & 1 & 1 & 1 & 1 & 1 & 1 & 1 & 1 & 1 & 1 \\
\hline Planariidae & Plan & 11 & 0 & 0 & 1 & 1 & 0 & 1 & 1 & 0 & 1 & 0 & 0 \\
\hline
\end{tabular}


Anexo 1. Continuación. Continuation.

TAXON

Planorbidae

Platycnemididae

Pleidae

Polycentropodidae

Polymitarcidae

Potamanthidae

Prosopistomatidae

Psychodidae

Psychomyiidae

Ptychopteridae

Pyralidae

Rhagionidae

Rhyacophilidae

Sciomyzidae

Sericostomatidae

Sialidae

Simuliidae

Siphlonuridae

Sphaeriidae

Stratiomyidae

Syrphidae

Tabanidae

Taeniopterygidae

Thaumaleidae

Thiaridae

Tipulidae

Valvatidae

Veliidae

Viviparidae

Código Adra Aguas Almanzora Besos Guadalfeo Júcar Llobregat Mijares Pollença Segura Sóller Turia

\begin{tabular}{|c|c|c|c|c|c|c|c|c|c|c|c|c|}
\hline $\mathrm{N}^{\circ}$ total de familias & 85 & 64 & 74 & 93 & 76 & 84 & 94 & 71 & 60 & 102 & 51 & 77 \\
\hline $\mathrm{N}^{\circ}$ de muestras & 39 & 43 & 79 & 98 & 121 & 69 & 102 & 33 & 32 & 109 & 17 & 44 \\
\hline
\end{tabular}

